\title{
Strong pure infiniteness of crossed products
}

\author{
E. KIRCHBERG $\dagger$ and A. SIERAKOWSKI $\$$ \\ $\dagger$ Institut für Mathematik, Humboldt Universität zu Berlin, D-10099 Berlin, Germany \\ (e-mail: kirchbrg@mathematik.hu-berlin.de) \\ $\ddagger$ School of Mathematics \& Applied Statistics, University of Wollongong, Wollongong, \\ NSW 2522, Australia \\ (e-mail: asierako@uow.edu.au)
}

(Received 5 August 2015 and accepted in revised form 12 February 2016)

\begin{abstract}
Consider an exact action of a discrete group $G$ on a separable $C^{*}$ algebra $A$. It is shown that the reduced crossed product $A \rtimes_{\sigma, \lambda} G$ is strongly purely infinite-provided that the action of $G$ on any quotient $A / I$ by a $G$-invariant closed ideal $I \neq A$ is element-wise properly outer and that the action of $G$ on $A$ is $G$ separating (cf. Definition 5.1). This is the first non-trivial sufficient general criterion for strong pure infiniteness of reduced crossed products of $C^{*}$-algebras $A$ that are not $G$-simple. In the case $A=\mathrm{C}_{0}(X)$, the notion of a $G$-separating action corresponds to the property that two compact sets $C_{1}$ and $C_{2}$, that are contained in open subsets $C_{j} \subseteq U_{j} \subseteq X$, can be mapped by elements $g_{1}, g_{2}$ of $G$ onto disjoint sets $\sigma_{g_{j}}\left(C_{j}\right) \subseteq U_{j}$, but satisfy not necessarily the contraction property $\sigma_{g_{j}}\left(U_{j}\right) \subseteq \overline{U_{j}}$. A generalization of strong boundary actions on compact spaces to non-unital and non-commutative $C^{*}$-algebras $A$ (cf. Definition 7.1) is also introduced. It is stronger than the notion of $G$-separating actions by Proposition 7.6, because $G$-separation does not imply $G$-simplicity and there are examples of $G$-separating actions with reduced crossed products that are stably projection-less and non-simple.
\end{abstract}

\section{Contents}

1 Introduction 221

2 Preliminaries $\quad 222$

3 Some used definitions and results $\quad 224$

4 Properly outer actions and ideal structure $\quad 225$

5 Strongly purely infinite crossed products 230

6 The case of commutative $C^{*}$-algebras $\quad 232$

7 Strong boundary actions versus $G$-separating actions 234

$\begin{array}{ll}\text { Acknowledgements } & 242\end{array}$

References $\quad 242$ 


\section{Introduction}

In this paper we carry on the study of $C^{*}$-dynamical systems with applications in classification via equivariant $K K$-theory. It was shown by the first named author that for any two stable separable nuclear strongly purely infinite $C^{*}$-algebras, both with primitive ideal space isomorphic to the same $T_{0}$-space $X$, the algebras are isomorphic if and only if they are $K K_{X}$-equivalent. Since until now it is unknown if non-simple nuclear purely infinite $C^{*}$-algebras are also strongly purely infinite, the verification of pure infiniteness is not suitable for classification purposes. It is however far from understood when $C^{*}$-algebra crossed products $A \rtimes_{\sigma, \lambda} G$ associated to $C^{*}$-dynamical systems are strongly purely infinite in terms of properties of the action $\sigma$, in particular in the non-simple case. Our main focus of this work is such characterization for crossed products that are either simple or more generally contain ideals coming from arbitrary $G$-invariant ideals of the algebra $A$ on which the group $G$ acts.

We begin (in §2) by introducing crossed products and by providing the notation used throughout the paper.

In $\S 4$, we look at results related to the ideal structure of crossed products. It was shown in [22] that residually properly outer (Definition 4.1) and exact (Definition 4.5) actions $\sigma: G \rightarrow \operatorname{Aut}(A)$ on a separable $C^{*}$-algebra $A$ have the property that the lattice of (closed) ideals of the reduced crossed product $A \rtimes_{\sigma, \lambda} G$ is naturally isomorphic to the lattice of $G$-invariant ideals of $A$ (by the map $I \mapsto A \cap I$ ). We refine this result by showing that for any exact and residually properly outer action $\sigma$ of a discrete group $G$ on a separable or commutative $C^{*}$-algebra $A$, the set $A_{+}$is a filling family (Definition 3.3) for $A \rtimes_{\sigma, \lambda} G$ (which implies that $I \mapsto A \cap I$ is a bijective map from the lattice of ideals in $A \rtimes_{\sigma, \lambda} G$ onto the lattice of $G$-invariant ideals in $A$; see Remark 3.5 for details).

In $\S 5$, we introduce the notion of $G$-separating actions (Definition 5.1). We show in Theorem 5.3 that for any exact and residually properly outer action $\sigma$ of a discrete group $G$ on a separable or commutative $C^{*}$-algebra $A$ and for any filling family $\mathcal{F} \subseteq A_{+}$, the crossed product $A \rtimes_{\sigma, \lambda} G$ is strongly purely infinite if and only if $\mathcal{F}$ has the diagonalization property (Definition 3.9) in $A \rtimes_{\sigma, \lambda} G$. Applying the results in the related paper [14], we obtain (in Proposition 5.5) an equivalent characterization of $G$-separating actions, from which we can deduce that $A_{+}$has the diagonalization property whenever the action on $A$ is $G$-separating. By using [14] once again, we prove our main result.

THEOREM 1.1. Suppose that $(A, G, \sigma)$ is a $C^{*}$-dynamical system, where $G$ is discrete and $A$ is separable or commutative.

If the action $\sigma$ of $G$ on $A$ is exact (Definition 4.5), residually properly outer (Definition 4.1) and G-separating (Definition 5.1), then $A \rtimes_{\sigma, \lambda} G$ is strongly purely infinite.

In $\S 6$, we look at actions on commutative $C^{*}$-algebras. Here we characterize the notion of $G$-separating action purely in terms of the underlying geometry. More specifically, we consider actions $\alpha$ of a discrete group $G$ on a locally compact Hausdorff space $X$, and denote by $\sigma$ the induced action on $A:=\mathrm{C}_{0}(X)$. We show (in Lemma 6.1) that the action of $G$ on $A$ is $G$-separating if and only if the action satisfies the following condition: for every two open compact sets $K_{1}, K_{2}$ contained in open subsets $K_{j} \subseteq U_{j} \subseteq X$, there 
exist $g_{j} \in G$ such that $\alpha_{g_{j}}\left(K_{j}\right) \subseteq U_{j}$, and $\alpha_{g_{1}}\left(K_{1}\right) \cap \alpha_{g_{2}}\left(K_{2}\right)=\emptyset$. This result is what motivates the choice of our terminology ' $G$-separating'. Notice that it does not imply that $\alpha_{g_{j}}\left(U_{j}\right) \subseteq \overline{U_{j}}$. As a consequence of this characterization, we obtain in Corollary 6.2 a topological sufficiency criterion for the strong pure infiniteness of the crossed product $\mathrm{C}_{0}(X) \rtimes_{\sigma, \lambda} G$ in terms of a condition on the action $\alpha$ of $G$ on $X$.

In the final $\S 7$, we consider actions that produce simple and strongly purely infinite crossed products. We introduce (Definitions 7.1 and 7.2) the notions of $n$-majorizing $(n \geq 1)$ and $n$-covering actions $(n \geq 2)$, the latter for actions on unital $C^{*}$-algebras. These two notions generalize and refine results on simple purely infinite crossed products in $[\mathbf{1 0}, \mathbf{1 7}]$, where the notions of strong boundary actions (Definition 3.1) and $n$-filling actions (Definition 3.2) were introduced. We prove in Remark 7.7 that our notions are weaker: any $n$-filling action on a unital $C^{*}$-algebra $A$ is $n$-covering and, for any action $\alpha$ on a compact space $X$ with more than two points (on which strong boundary actions are defined), the action $\alpha$ is a strong boundary action if and only if its adjoint action $\sigma$ on $\mathrm{C}(X)$ is 1 -majorizing. Both our notions are $G$-simple. Therefore, we call the 1-majorizing actions on not-necessarily unital or commutative $C^{*}$-algebras also strong boundary actions. Despite our weaker assumptions, we are able to prove the following result.

THEOREM 1.2. Suppose that the $C^{*}$-dynamical system $(A, G, \sigma)$ with discrete $G$ is $n$-majorizing (Definition 7.1) for some $n \geq 1$ or $n$-covering (Definition 7.2) for some $n \geq 2$, the latter if $A$ is unital. If the action $\sigma$ is element-wise properly outer (Definition 4.1) and $A$ is separable or commutative, then $A \rtimes_{\sigma, \lambda} G$ is simple strongly purely infinite.

In $§ 7$, we also look at how the different properties relate to each other. In Lemma 7.3, we show that each $n$-covering action (for $n \geq 2$ ) on a unital $C^{*}$-algebra $A$ is $n$-majorizing, and the latter properly (for $n \geq 1$ ) implies that the action is $(n+1)$-covering. In Proposition 7.6, we prove that that any 1 -majorizing action on a non-unital $C^{*}$-algebra $A$ is automatically $G$-separating. In Remark 7.7, we prove that any action on a unital commutative $C^{*}$-algebra $A$ is $n$-filling if and only if it is $n$-covering and, for $n=2$, this is again equivalent to a strong boundary (i.e., 1-majorizing) action.

We end with a number of remarks, including a proof of the fact that our notions of $G$-separating, $n$-majorizing and $n$-covering actions can be expressed in terms of projections when $A$ has real rank zero (see Remark 7.9).

We hope that the study of crossed products-even those for actions of amenable discrete groups on locally compact Polish spaces_ can help to detect possible differences between strong and weak pure infiniteness. This paper is a very first step in this direction, and gives a sufficient criterion by conditions on the action that implies strong pure infiniteness of reduced crossed products.

\section{Preliminaries}

We let $A_{+}$denote the set of positive elements in a $C^{*}$-algebra $A$. We denote the positive and negative parts of a self-adjoint element $a \in A$ by $a_{+}:=(|a|+a) / 2 \in A_{+}$and $a_{-}:=$ $(|a|-a) / 2) \in A_{+}$, where $|a|:=\left(a^{*} a\right)^{1 / 2}$. If $a \in A_{+}$, then $(a-\varepsilon)_{+}$, the positive part of $a-\varepsilon 1$ in $\mathcal{M}(A)$, is again in $A_{+}$. Here $\mathcal{M}(A)$ is the multiplier algebra of $A$. This notation will be used also for functions $f: \mathbb{R} \rightarrow \mathbb{R}$; then e.g. $(f-\varepsilon)_{+}(\xi)=\max (f(\xi)-\varepsilon, 0)$. 
A subset $\mathcal{F} \subseteq A_{+}$is invariant under $\varepsilon$-cut-downs if for each $a \in \mathcal{F}$ and $\varepsilon \in(0,\|a\|)$ we have $(a-\varepsilon)_{+} \in \mathcal{F}$. The minimal unitalization of $A$ is denoted $\tilde{A}$. Restriction of a map $f$ to $X$ is denoted $f \mid X$. We let $\mathrm{C}_{c}(0, \infty]_{+}$denote the set of all non-negative continuous functions $\varphi$ on $[0, \infty)$ with $\varphi \mid[0, \eta]=0$ for some $\eta \in(0, \infty)$ such that $\lim _{t \rightarrow \infty} \varphi(t)$ exists.

Remarks 2.1.

(i) Suppose that $a, b \in A_{+}$and $\varepsilon>0$ satisfy $\|a-b\|<\varepsilon$. Then the positive part $(b-\varepsilon)_{+} \in A$ of $(b-\varepsilon \cdot 1) \in \mathcal{M}(A)$ can be decomposed into $d^{*} a d=(b-\varepsilon)_{+}$with some contraction $d \in A[\mathbf{1 3}$, Lemma 2.2].

(ii) Let $\tau \in[0, \infty)$ and $0 \leq b \leq a+\tau \cdot 1$ (in $\mathcal{M}(A)$ ); then for every $\varepsilon>\tau$ there is a contraction $f \in A$ such that $(b-\varepsilon)_{+}=f^{*} a_{+} f$. (See [13, Lemma 2.2] and $[3, \S 2.7]$.)

We abbreviate $C^{*}$-dynamical systems by $(A, G, \sigma)$ with discrete groups $G$. We denote by $e$ the unit of $G$ and consider only closed and two-sided ideals of $A$. The reduced (respectively the full) crossed product associated to $(A, G, \sigma)$ is denoted by $A \rtimes_{\sigma, \lambda} G$ (respectively $A \rtimes_{\sigma} G$ ). The norm on $A \rtimes_{\sigma, \lambda} G$ will be sometimes written as $\|\cdot\|_{\lambda}$ if it is necessary to distinguish it from other norms. We let $\mathcal{I}(A)$ denote the lattice of ideals in a $C^{*}$-algebra $A$. The map $\eta: A \rightarrow A \rtimes_{\sigma} G$ means the natural embedding into the full crossed product. Let $\pi_{\lambda}: A \rtimes_{\sigma} G \rightarrow A \rtimes_{\sigma, \lambda} G$ be the natural epimorphism. We will sometimes suppress the canonical inclusion maps $\eta: A \rightarrow A \rtimes_{\sigma} G$ and $\pi_{\lambda} \circ \eta: A \rightarrow$ $A \rtimes_{\sigma, \lambda} G$. Let $U$ denote the canonical unitary representation $U: G \rightarrow \mathcal{M}\left(A \rtimes_{\sigma} G\right)$. Notice here that the linear $\operatorname{span} \eta(A) U(G)$ is a dense *-subalgebra of $A \rtimes_{\sigma} G$. We denote by $U_{\lambda}: G \rightarrow \mathcal{M}\left(A \rtimes_{\sigma, \lambda} G\right)$ the regular representation for some more precise explanations. The same happens for the reduced crossed product with $\eta_{\lambda}:=\pi_{\lambda} \circ \eta$ in place of $\eta$.

The set $\mathrm{C}_{c}(G, A)$ consists of the maps $f: G \rightarrow A$ with finite support $F:=G \backslash f^{-1}(0)$. There is a natural linear embedding of $\mathrm{C}_{c}(G, A)$ into $A \rtimes_{\sigma} G$ by canonical identification of $f: G \rightarrow A$ (of finite support) with an element of $A \rtimes_{\sigma} G$ : let $F \subseteq G$ be a finite subset with $f(g)=0$ for $g \notin F$. Then $f$ will be identified with the element $\sum_{g \in F} \eta\left(a_{g}\right) U(g)$ of $A \rtimes_{\sigma} G$, where $a_{g}:=f(g)$. In this way, $\mathrm{C}_{c}(G, A)$ becomes a *-subalgebra of $A \rtimes_{\sigma} G$ that unitally contains $A$. The natural $C^{*}$-morphism $\pi_{\lambda}: A \rtimes_{\sigma} G \rightarrow A \rtimes_{\sigma, \lambda} G$ is faithful on $\mathrm{C}_{c}(G, A)$, and we do not distinguish between

$$
\pi_{\lambda}\left(\sum_{g \in F} \eta\left(a_{g}\right) U(g)\right)=\sum_{g \in F} \eta_{\lambda}\left(a_{g}\right) U_{\lambda}(g)
$$

and $\sum_{g \in F} \eta\left(a_{g}\right) U(g)$. In particular, $\eta(a) \in A \rtimes_{\sigma} G$ and $\eta_{\lambda}(a) \in A \rtimes_{\sigma, \lambda} G$ will be denoted simply by $a \in A$, and $U_{\lambda}(g)$ might be denoted $U(g)$.

We now recall the conditional expectation $E: A \rtimes_{\sigma} G \rightarrow \eta(A) \cong A$ : the map $E_{\text {alg }}$ : $\mathrm{C}_{c}(G, A) \rightarrow A, \sum_{g \in F} a_{g} U(g) \mapsto a_{e}$, extends by continuity to a faithful conditional expectation $E_{\lambda}: A \rtimes_{\sigma, \lambda} G \rightarrow A$. In particular, $E_{\lambda}$ is a completely positive contraction, $E_{\lambda}\left(A \rtimes_{\sigma, \lambda} G\right)=A$, and $E_{\lambda}(b)=0$ imply $b=0$ for $b \in\left(A \rtimes_{\sigma, \lambda} G\right)_{+}$. Since $A$ is also contained in its full crossed product $A \rtimes_{\sigma} G$, we can use the natural epimorphism $A \rtimes_{\sigma}$ $G \rightarrow A \rtimes_{\sigma, \lambda} G$ to define $E$ by $E:=E_{\lambda} \circ \pi_{\lambda}$ as a (not necessarily faithful) conditional expectation from $A \rtimes_{\sigma} G$ onto its $C^{*}$-subalgebra $A$. 
3. Some used definitions and results

For the convenience of the reader, we include here a few used definitions and results that are frequently cited throughout this paper. The paper [14] is available as a preprint.

Definition 3.1. [17] Let $\alpha$ be an action of a discrete group $G$ on a compact space $X$ with at least three points. The action $\alpha$ is a strong boundary action if for every pair $U$ and $V$ of non-empty open subsets of $X$ there exists $t \in G$ such that $\alpha_{t}(X \backslash U) \subseteq V$.

Definition 3.2. [10] An action $\sigma$ of a discrete group $G$ on a unital $C^{*}$-algebra $A$ is $n$-filling $(n \geq 2)$ if, for all $b_{1}, \ldots, b_{n} \in A_{+}$, with $\left\|b_{j}\right\|=1$ for each $j$, and all $\varepsilon>0$, there exist $g_{1}, \ldots, g_{n} \in G$ such that $\sum_{j=1}^{n} \sigma_{g_{j}}\left(b_{j}\right) \geq 1-\varepsilon$.

Definition 3.3. [14] Let $\mathcal{F}$ be a subset of $A_{+}$. The set $\mathcal{F}$ is a filling family for $A$ if $\mathcal{F}$ satisfies the following equivalent conditions (i) and (ii).

(i) For every $a, b, c \in A$ with $0 \leq a \leq b \leq c \leq 1$, with $a b=a \neq 0$ and $b c=b$, there exist $z_{1}, z_{2}, \ldots, z_{n} \in A$ and $d \in A$ with $z_{j}\left(z_{j}\right)^{*} \in \mathcal{F}$ such that $e c=e$ and $d^{*} e d=a$ for $e:=z_{1}^{*} z_{1}+\cdots+z_{n}^{*} z_{n}$.

(ii) For every hereditary $C^{*}$-subalgebra $D$ of $A$ and every primitive ideal $I$ of $A$ with $D \nsubseteq I$, there exist $f \in \mathcal{F}$ and $z \in A$ with $z^{*} z \in D$ and $z z^{*}=f \notin I$.

Lemma 3.4. [14] Suppose that $A \subseteq B$ is a $C^{*}$-subalgebra of $B$ and $\mathcal{F} \subseteq A_{+}$is a subset of $A_{+}$. If $\mathcal{F}$ is filling for $A$, and $A_{+}$is filling for $B$, then $\mathcal{F}$ is a filling family for $B$.

Remark 3.5. [14] Let $A \subseteq B$ be $C^{*}$-algebras and $\mathcal{F} \subseteq A_{+}$. If $\mathcal{F}:=A_{+} \subseteq B$ is filling for $B$, then the map $I \in \mathcal{I}(B) \mapsto I \cap A \in \mathcal{I}(A)$ is injective, i.e., $A$ separates the closed ideals of $B$.

Definition 3.6. [14] A $C^{*}$-algebra $A$ is strongly purely infinite (for short: s.p.i.) if, for every $a, b \in A_{+}$and $\varepsilon>0$, there exist elements $s, t \in A$ such that

$$
\left\|s^{*} a^{2} s-a^{2}\right\|<\varepsilon, \quad\left\|t^{*} b^{2} t-b^{2}\right\|<\varepsilon \quad \text { and } \quad\left\|s^{*} a b t\right\|<\varepsilon .
$$

Remark 3.7. [14] A $C^{*}$-algebra $A$ is strongly purely infinite if and only if for every $a, b \in A_{+}, c \in A$ and $\varepsilon>0$, there exist contractions $s, t \in A$ such that

$$
\left\|s^{*} a s-a\right\|<\varepsilon, \quad\left\|t^{*} b t-b\right\|<\varepsilon \quad \text { and } \quad\left\|s^{*} c t\right\|<\varepsilon .
$$

Definition 3.8. [14] Let $\mathcal{S} \subseteq A$ be a multiplicative sub-semigroup of a $C^{*}$-algebra $A$ and $\mathcal{C} \subseteq A$ a subset of $A$. An $n$-tuple $\left(a_{1}, \ldots, a_{n}\right)$ of positive elements in $A$ has the matrix diagonalization property with respect to $\mathcal{S}$ and $\mathcal{C}$ if, for every $\left[a_{i j}\right] \in M_{n}(A)_{+}$with $a_{j j}=a_{j}$ and $a_{i j} \in \mathcal{C}$ (for $i \neq j$ ) and $\varepsilon_{j}>0, \tau>0$, there are elements $s_{1}, \ldots, s_{n} \in \mathcal{S}$ with

$$
\left\|s_{j}^{*} a_{j j} s_{j}-a_{j j}\right\|<\varepsilon_{j} \quad \text { and } \quad\left\|s_{i}^{*} a_{i j} s_{j}\right\|<\tau \quad \text { for } i \neq j .
$$

If $\mathcal{S}=\mathcal{C}=A$, then this is the matrix diagonalization property of $\left(a_{1}, \ldots, a_{n}\right)$ as defined in [13, Definition 5.5], and we say that $\left(a_{1}, \ldots, a_{n}\right)$ has matrix diagonalization (in $A$ ).

Definition 3.9. [14] Let $\mathcal{F}$ be a subset of $A_{+}$. The family $\mathcal{F}$ has the (matrix) diagonalization property (in $A$ ) if each finite sequence $a_{1}, \ldots, a_{n} \in \mathcal{F}$ has the matrix diagonalization property (in $A$ ) of Definition 3.8. 
Lemma 3.10. [14] Suppose that $\mathcal{F} \subseteq A_{+}$is invariant under $\varepsilon$-cut-downs, i.e., that for each $a \in \mathcal{F}$ and $\varepsilon \in(0,\|a\|)$, we have $(a-\varepsilon)_{+} \in \mathcal{F}$.

Then the family $\mathcal{F}$ has the matrix diagonalization property if and only if each pair of elements in $\mathcal{F}$ has the matrix diagonalization property of Definition 3.8.

(It was Lemma 3.12 in the first version of this paper.)

LEMMA 3.11. [14] Let $\varepsilon_{0}>0$ and non-empty subsets $\mathcal{F} \subseteq A_{+}, \mathcal{C} \subseteq A$ be given, and let $\mathcal{S} \subseteq A$ be a (multiplicative) sub-semigroup of A that satisfies $s_{2}^{*} \mathcal{C} s_{1} \subseteq \mathcal{C}$ for all $s_{1}, s_{2} \in \mathcal{S}$. Suppose that the following properties hold.

(i) For every $\varepsilon_{0}>\delta>0$, the pair $\left(\left(a_{1}-\delta\right)_{+},\left(a_{2}-\delta\right)_{+}\right)$has the matrix diagonalization property with respect to $\mathcal{S}$ and $\mathcal{C}$ of Definition 3.8 .

(ii) $\varphi\left(a_{1}\right) c \varphi\left(a_{2}\right) \in \mathcal{C}$ for each $c \in \mathcal{C}$ and $\varphi \in \mathrm{C}_{c}(0, \infty]_{+}$.

(iii) $\varphi\left(a_{1}\right) s, \varphi\left(a_{2}\right) s \in \mathcal{S}$ for each $s \in \mathcal{S}$ and $\varphi \in \mathrm{C}_{c}(0, \infty]_{+}$.

Then, for every $c \in \overline{\operatorname{span}(\mathcal{C})}, a_{1}, a_{2} \in \mathcal{F}, \varepsilon_{0} / 2 \geq \varepsilon>0$ and $\tau>0$, there exist $s_{1}, s_{2} \in \mathcal{S}$ that fulfil $\left\|s_{j}\right\|^{2} \leq 2\left\|a_{j}\right\| / \varepsilon$ and

$$
\left\|s_{1}^{*} a_{1} s_{1}-a_{1}\right\|<\varepsilon, \quad\left\|s_{2}^{*} a_{2} s_{2}-a_{2}\right\|<\varepsilon \quad \text { and } \quad\left\|s_{1}^{*} c s_{2}\right\|<\tau .
$$

THEOREM 3.12. [14] The minimal tensor product of a strongly purely infinite $C^{*}$-algebra and an exact $C^{*}$-algebra is strongly purely infinite.

THEOREM 3.13. [14] Suppose that $A_{+}$contains a filling family $\mathcal{F}$ that has the diagonalization property (in A). Then A is strongly purely infinite.

\section{Properly outer actions and ideal structure}

In this section we look at conditions on a $C^{*}$-dynamical system $(A, G, \sigma)$ ensuring that the set $A_{+}$is a filling family for $A \rtimes_{\sigma, \lambda} G$ in the sense of Definition 3.3. This implies in particular a one-to-one correspondence between ideals of $A \rtimes_{\sigma, \lambda} G$ and $G$-invariant ideals of $A$, but (as we shall see) also applies to the verification of when a crossed product is strongly purely infinite. A crucial ingredient of the following consideration is that the automorphisms $\sigma_{t}$ for $t \neq e$ are all properly outer. We recall the definition below.

Definition 4.1. Suppose that $(A, G, \sigma)$ is a $C^{*}$-dynamical system and that $G$ is discrete. The action $\sigma$ will be called element-wise properly outer if, for each $g \in G \backslash\{e\}$, the automorphism $\sigma_{g}$ of $A$ is properly outer in the sense of [6, Definition 2.1], i.e., $\left\|\sigma_{g} \mid I-\operatorname{Ad}(U)\right\|=2$ for any $\sigma_{g}$-invariant non-zero ideal $I$ of $A$ and any unitary $U$ in the multiplier algebra $\mathcal{M}(I)$ of $I$. See also [18, Theorem 6.6(ii)].

We call here an action $\sigma$ residually properly outer if, for every $G$-invariant ideal $J \neq A$ of $A$, the induced action $[\sigma]_{J}$ of $G$ on $A / J$ is element-wise properly outer.

\section{Remarks 4.2.}

(i) Notice that element-wise proper outerness passes to subgroups, i.e., for each subgroup $H \subseteq G$, the system $(A, H, \sigma \mid H)$ is element-wise properly outer on $A$ if $(A, G, \sigma)$ is element-wise properly outer. But residual proper outerness does not necessarily pass to subgroups. The system $(A, H, \sigma \mid H)$ is not necessarily residually properly outer if $(A, G, \sigma)$ is residual proper outer, because possibly there could be more $H$-invariant ideals than $G$-invariant ideals of $A$. 
(ii) If $A$ is non-commutative, then topological freeness of $(A, G, \sigma)$ in the sense of [1, Definition 1] is - at least formally—stronger than the assumption of element-wise proper outerness of $(A, G, \sigma)$ in Definition 4.1 (cf. [1, Proposition 1]). We do not know examples where they actually differ. Thus, for non-commutative $A$, 'essential freeness' of the corresponding action of $G$ on $\widehat{A}$ in the sense of [22, Definition 1.17] (inspired by [20, Definition 4.8]) is-formally_-stronger than our residual proper outerness of $(A, G, \sigma)$.

(iii) If $G$ is countable and acting on $\mathrm{C}_{0}(X)$, one can show-using the Baire property of $X$-that element-wise proper outerness is the same as the requirement (for the action $\alpha$ of $G$ on $X$ with $\left.\sigma_{g}(f):=f \circ \alpha_{g^{-1}}\right)$ that points with trivial fix-point subgroup (trivial isotropy) are dense in $X$, i.e., [22, Definition 1.17] holds. We can reformulate this as: stability subgroups of non-empty open subsets are trivial.

Remark 4.3. We recall [18, Lemma 7.1.] (cf. also [16, Lemma 3.2]).

If $\alpha_{1}, \alpha_{2}, \ldots, \alpha_{n}$ are properly outer automorphisms of a separable $C^{*}$-algebra $A$, there is, for each $a_{0}, a_{1}, a_{2}, \ldots, a_{n} \in \widetilde{A}$, with $0 \neq a_{0} \geq 0$, and each $\varepsilon>0$, an element $x \in A_{+}$ with $\|x\|=1$ such that

$$
\left\|x a_{0} x\right\|>\left\|a_{0}\right\|-\varepsilon, \quad\left\|x a_{i} \alpha_{j}(x)\right\|<\varepsilon, \quad 1 \leq i, j \leq n .
$$

If $A$ is commutative, i.e., $A \cong \mathrm{C}_{0}(X)$ for $X=\widehat{A} \subseteq A^{*}$, then it is not necessary to suppose that $A$ is separable in the above-quoted lemma of Olesen and Pedersen (compare also [7]): an automorphism $\sigma \in \operatorname{Aut}(A)$ is properly outer if and only if, for every open subset $\emptyset \neq U \subseteq X$, there exists $y \in U$ with $\widehat{\sigma}(y) \neq y$. Thus, for every finite set $S \subseteq \operatorname{Aut}(A)$ of properly outer automorphisms, every non-empty open subset $U \subseteq X$ contains a non-empty open subset $V \subseteq U$ with $\widehat{\sigma}(V) \cap V=\emptyset$ for all $\sigma \in S$. If one takes $U:=a_{0}^{-1}\left(\left\|a_{0}\right\|-\varepsilon, \infty\right)$ and non-empty $V \subseteq U$ as above, then each $x \in \mathrm{C}_{0}(X)$ with $\|x\|=1$ and support in $V$ satisfies $\left\|x a_{0} x\right\|>\left\|a_{0}\right\|-\varepsilon$ and $x \sigma(x)=0$ for $\sigma \in S$.

The following Lemma 4.4 is a suitable modification of proofs of [18, Lemma 7.1 and Theorem 7.2]. It has been proved in [1] under the stronger assumption that the action $\sigma$ is topologically free, and part (iii) has been shown in [11, Theorem 4.1] even to be equivalent to the topological freeness of the action if $A$ is commutative and unital and $G$ is amenable. Compare also Corollary 4.9 for a 'residual' version.

Lemma 4.4. Suppose that $A$ is separable or commutative and that the action of $G$ on $A$ is element-wise properly outer.

(i) For every $b \in\left(A \rtimes_{\sigma} G\right)_{+}$with $E(b) \neq 0$ and $\varepsilon>0$, there exists $x \in A_{+}$satisfying

$$
\|x\|=1, \quad\|x b x-x E(b) x\|<\varepsilon, \quad\|x E(b) x\|>\|E(b)\|-\varepsilon .
$$

This holds also for $b \in\left(A \rtimes_{\sigma, \lambda} G\right)_{+}$and $E_{\lambda}$ in place of $E$.

(ii) If $h: A \rtimes_{\sigma} G \rightarrow \mathcal{L}(\mathcal{H})$ is a *-representation such that $h \mid A$ is faithful, then $\|h(b)\| \geq\|E(b)\|$ for all $b \in\left(A \rtimes_{\sigma} G\right)_{+}$.

In particular, the kernel of $h$ is contained in the kernel $I_{\lambda}$ of the natural epimorphism $\pi_{\lambda}: A \rtimes_{\sigma} G \rightarrow A \rtimes_{\sigma, \lambda} G$.

(iii) Every non-zero ideal of $A \rtimes_{\sigma, \lambda} G$ has non-zero intersection with $A$. 
Proof. (i) Let $b \in\left(A \rtimes_{\sigma} G\right)_{+}$with $E(b) \neq 0$, and $\varepsilon>0$.

Let $a_{0}:=E(b)$. Since $\mathrm{C}_{c}(G, A)$ is dense in $A \rtimes_{\sigma} G$, there exists $a^{\prime}=c_{0}+$ $\sum_{j=1}^{m} a_{j} U\left(g_{j}\right) \in \mathrm{C}_{c}(G, A)$ with $g_{i}^{-1} g_{j} \neq e$ and $g_{j} \neq e$ for $i \neq j \in\{1, \ldots, m\}$, and $\left\|a^{\prime}-b\right\|<\varepsilon / 6$. Since $E$ is a contraction, it follows that $\|b-a\|<\varepsilon / 3$ and $E(a)=$ $a_{0}=E(b)$ for $g_{0}:=e$ and $a:=a_{0}+\left(a^{\prime}-c_{0}\right)=\sum_{j=0}^{m} a_{j} U\left(g_{j}\right)$. By [18, Lemma 7.1] and Remark 4.3, there exists $x \in A_{+}$with $\|x\|=1,\|x E(a) x\|>\|E(a)\|-\varepsilon / 3 m$ and $\left\|x a_{j} \sigma_{g_{j}}(x)\right\|<\varepsilon / 3 m$ for $g_{j} \neq e, j=1, \ldots, m$. In particular,

$$
\|x E(b) x\|=\|x E(a) x\|>\|E(a)\|-\varepsilon=\|E(b)\|-\varepsilon .
$$

Since $\left\|x a_{j} U\left(g_{j}\right) x\right\|=\left\|x a_{j} \sigma_{g_{j}}(x)\right\|$, we get in $A \rtimes_{\sigma} G$ that

$$
\|x(a-E(a)) x\| \leq \sum_{g_{j} \neq e}\left\|x a_{j} \sigma_{g_{j}}(x)\right\| \leq \varepsilon / 3 .
$$

Thus, in the full crossed product $A \rtimes_{\sigma} G$, we have

$$
\|x b x-x E(b) x\| \leq\|x(b-a) x\|+\|x(a-E(a)) x\|+\|x(E(a)-E(b)) x\|<\varepsilon .
$$

The same arguments work for $b \in\left(A \rtimes_{\sigma, \lambda} G\right)_{+}$and $E_{\lambda}$ in place of $E$.

(ii) The restriction of $h$ to $A \subseteq A \rtimes_{\sigma} G$ is faithful and hence $\|h(a)\|=\|a\|$ for all $a \in A$. Let $b \in\left(A \rtimes_{\sigma} G\right)_{+}$be given. If $E(b)=0$, then $\|h(b)\| \geq\|E(b)\|$. If $E(b) \neq 0$, then select $x \in A_{+}$as in (i). It follows that $\|h(x E(b) x)\|=\|x E(b) x\| \geq\|E(b)\|-\varepsilon$. On the other hand, $\|h(b)\| \geq\|h(x) h(b) h(x)\|=\|h(x b x)\|$ and $\varepsilon>\|x b x-x E(b) x\| \geq$ $\|h(x b x)-h(x E(b) x)\|$. Thus, $\|h(b)\|+\varepsilon \geq\|h(x E(b) x)\|$, and $\|h(b)\|+2 \varepsilon \geq\|E(b)\|$ for all $\varepsilon>0$.

Since $E=E_{\lambda} \circ \pi_{\lambda}$, we have $b \in\left(A \rtimes_{\sigma} G\right)_{+}$and $E(b)=0$ implies that $b$ is contained in the kernel of $\pi_{\lambda}: A \rtimes_{\sigma} G \rightarrow A \rtimes_{\sigma, \lambda} G$. In particular, if $h: A \rtimes_{\sigma} G \rightarrow \mathcal{L}(\mathcal{H})$ is any *-representation with $\|h(b)\| \geq\|E(b)\|$ for all $b \in\left(A \rtimes_{\sigma} G\right)_{+}$, then the kernel $h^{-1}(0)$ of $h$ is contained in the kernel of the natural epimorphism $\pi_{\lambda}: A \rtimes_{\sigma} G \rightarrow A \rtimes_{\sigma, \lambda} G$.

(iii) Let $I$ be a closed ideal of $A \rtimes_{\sigma, \lambda} G$ with $I \cap A=\{0\}$, consider $\left(A \rtimes_{\sigma, \lambda} G\right) / I$ as a $C^{*}$-subalgebra of some $\mathcal{L}(\mathcal{H})$ and let $h: A \rtimes_{\sigma} G \rightarrow \mathcal{L}(\mathcal{H})$ be the corresponding representation with kernel $h^{-1}(0)=J:=\pi_{\lambda}^{-1}(I) \supseteq \pi_{\lambda}^{-1}(0)$. Then $h$ is faithful on $A$ and, therefore, satisfies $\pi_{\lambda}^{-1}(0) \supseteq h^{-1}(0)$. It follows that $I=\pi_{\lambda}\left(h^{-1}(0)\right)=\{0\}$.

Definition 4.5. [22, Definition 1.2] Suppose that $(A, G, \sigma)$ is a $C^{*}$-dynamical system with locally compact $G$. The action $\sigma$ of $G$ on $A$ is exact if, for every $G$-invariant ideal $J$ in $A$, the sequence $0 \rightarrow J \rtimes_{\sigma \mid J, \lambda} G \rightarrow A \rtimes_{\sigma, \lambda} G \rightarrow A / J \rtimes_{[\sigma]_{J}, \lambda} G \rightarrow 0$ is short-exact.

\section{Remarks 4.6.}

(i) Recall that a locally compact group $G$ is exact if and only if every action $\sigma: G \rightarrow$ $\operatorname{Aut}(A)$ is exact. If $G$ is discrete, then this is equivalent to the exactness of the $C^{*}$ algebra $C_{\lambda}^{*}(G)$; cf. [15]. This applies to all amenable groups $G$, e.g. $G=\mathbb{Z}$. Under Definition 4.5, each minimal (=G-simple) action is exact. In particular, non-exact discrete groups can have exact (and faithful) actions.

(ii) Let $F$ denote the (small) Thompson group and $\rho: F \rightarrow \operatorname{Homeo}(\mathbb{R})$ the minimal action of $F$ (or only of its commutator subgroup $F^{\prime}$ ) on the real line $\mathbb{R}$ as described by Haagerup and Picioroaga in [9, Remark 2.5]. One can use $\rho$ to construct a 
$F$-separating, non-minimal and exact action $\alpha$ of $F$ (or $F^{\prime}$ ) on the disjoint union of two lines $X:=\mathbb{R} \cup(i+\mathbb{R}) \subseteq \mathbb{C}$ if one considers the restriction $\alpha(g):=\beta(g) \mid X$ to $X$ of the action $g \in F \rightarrow \beta(g)$ on $\mathbb{C}$ given by $\beta(g)(s+i t):=\rho(g)(s)+i t \dagger$. It is at present unknown whether the Thompson group $F$ is amenable or not; cf. [2, 8]. It is not even known if $F$ is exact.

(iii) It is not known if Gromov's examples of non-exact groups can have non-exact actions on commutative $C^{*}$-algebras. It is likely that it has to do with still missing non-trivial geometric conditions for $G$-actions on locally compact spaces $X$ that are equivalent to the exactness of the adjoint action $\sigma: G \rightarrow \operatorname{Aut}\left(\mathrm{C}_{0}(X)\right)$ given by $\sigma_{g}(f):=f \circ$ $\alpha_{g^{-1}}$. Therefore, we use the trivial and non-geometric definition and define $\alpha$ to be exact if its adjoint action $\sigma$ on $\mathrm{C}_{0}(X)$ is exact.

Remark 4.7. Combination of Lemma 4.4(iii) and of the exactness of an action $\sigma: G \rightarrow$ $\operatorname{Aut}(A)$ on a separable or commutative $C^{*}$-algebra $A$ shows that the lattice of (closed) ideals of the reduced crossed product $A \rtimes_{\sigma, \lambda} G$ is naturally isomorphic to the lattice of $G$-invariant ideals of $A$ (by the map $J \mapsto A \cap J$ ) if $\sigma$ is exact and residually proper outer. (See [22, Remark 2.23] for details.)

THEOREM 4.8. Let $(A, G, \sigma)$ be a $C^{*}$-dynamical system with discrete $G$ and separable or commutative A. If the action $\sigma$ of $G$ on $A$ is exact and residually properly outer, then the elements of $A_{+}$build a filling family for $A \rtimes_{\sigma, \lambda} G$ in the sense of Definition 3.3.

Proof. We show that for every hereditary $C^{*}$-subalgebra $D$ of $A \rtimes_{\sigma, \lambda} G$ and every (closed) ideal $I$ of $A \rtimes_{\sigma, \lambda} G$ with $D \nsubseteq I$, there exist $f \in A_{+}$and $z \in A \rtimes_{\sigma, \lambda} G$ such that $z^{*} z \in D$ and $z z^{*}=f \notin I$.

Suppose that $D$ is a hereditary $C^{*}$-subalgebra of $A \rtimes_{\sigma, \lambda} G$ and that $I$ is an ideal of $A \rtimes_{\sigma, \lambda} G$ with $D \nsubseteq I$. Let $J:=I \cap A$; then $J$ is a $G$-invariant ideal of $A$ with $J \rtimes_{\sigma \mid J, \lambda} G \subseteq I$ and $g \in G \mapsto\left[\sigma_{g}\right]_{J}$ is an element-wise properly outer action on $A / J$ by our assumptions on $\sigma$. We denote this action by $\alpha$, i.e., $\alpha_{g}(a+J):=\sigma_{g}(a)+J$.

By Remark 4.7, the exactness and residual proper outerness of $\sigma: G \rightarrow \operatorname{Aut}(A)$ allow a natural identification

$$
\left(A \rtimes_{\sigma, \lambda} G\right) / I=(A / J) \rtimes_{\alpha, \lambda} G .
$$

Since $D \nsubseteq I$ implies $D_{+} \nsubseteq I$, there exists $d \in D_{+}, d \notin I$. The epimorphism $\pi_{I}$ : $A \rtimes_{\sigma, \lambda} G \rightarrow\left(A \rtimes_{\sigma, \lambda} G\right) / I$ is equal to the quotient map $\pi^{J}$ from $A \rtimes_{\sigma, \lambda} G$ onto $(A / J) \rtimes_{\alpha, \lambda} G \cong\left(A \rtimes_{\sigma, \lambda} G\right) / I$ (under natural identifications). We denote the conditional expectation $E_{\lambda}:(A / J) \rtimes_{\alpha, \lambda} G \rightarrow A / J$ (temporarily) by $E$ and define

$$
b:=\pi_{I}(d) \quad \text { and } \quad \varepsilon:=\frac{1}{4}\|E(b)\|>0 .
$$

Lemma 4.4(i) gives an element $x \in(A / J)_{+}$such that

$$
\|x\|=1, \quad\|x b x-x E(b) x\|<\varepsilon, \quad\|x E(b) x\|>\|E(b)\|-\varepsilon=\frac{3}{4}\|E(b)\| .
$$

By Remark 2.1(i), there is a contraction $y \in(A / J) \rtimes_{\alpha, \lambda} G$ such that

$$
y^{*} x b x y=(x E(b) x-\varepsilon)_{+} \in(A / J)_{+} .
$$

$\dagger$ This action is not topologically free. 
Note that $y^{*} x b x y \neq 0$, because

$$
\left\|(x E(b) x-\varepsilon)_{+}\right\|=\|x E(b) x\|-\frac{1}{4}\|E(b)\|>\frac{1}{2}\|E(b)\|=2 \varepsilon .
$$

Since $\pi_{I}\left|A=\pi^{J}\right| A$ and $(x E(b) x-\varepsilon)_{+} \in(A / J)_{+}$, there exists $c \in A_{+}$such that $\pi^{J}(c)=$ $(x E(b) x-\varepsilon)_{+}$. Since $\pi^{J}\left(=\pi_{I}\right)$ is surjective, there exists a contraction $w \in A \rtimes_{\sigma, \lambda} G$ with $\pi^{J}(w)=x y$. We obtain that

$$
c=w^{*} d w+v
$$

for some $v \in I$. The set $\mathrm{C}_{c}(G, J)$ is dense in $I$, because $I=J \rtimes_{\sigma, \lambda} G$ and $G$ is discrete. This allows us to see that $J I J$ is dense in $I$. It follows that $\left\{e \in J_{+} \mid\|e\|<1\right\}$ is an approximate unit of $I$. In particular, there exists $e \in J_{+}$with $\|v-e v\|<\varepsilon$.

Let 1 denote the unit of $\widetilde{A} \rtimes_{\sigma, \lambda} G$; then $A \rtimes_{\sigma, \lambda} G$ is an ideal of $\widetilde{A} \rtimes_{\sigma, \lambda} G$. With $g:=(1-e) \in \widetilde{A}_{+},\|g\| \leq 1$, we get

$$
\left\|g w^{*} d w g-g c g\right\|=\|g v g\| \leq\|v-e v\|<\varepsilon=\frac{1}{4}\|E(b)\| .
$$

Since $g z g=z+e z e-(z e+e z)$ and $\pi_{I}(e)=\pi^{J}(e)=0$, we have $\pi_{I}(g z g)=\pi_{I}(z)$ for all $z \in A \rtimes_{\sigma, \lambda} G$.

By Remark 2.1(i), there exists a contraction $h \in A \rtimes_{\sigma, \lambda} G$ such that

$$
h^{*}\left(g w^{*} d w g\right) h=(g c g-\varepsilon)_{+} \in A_{+} .
$$

With $z:=\left(d^{1 / 2} w g h\right)^{*}$, we have that $z^{*} z \in D$ and $z z^{*}=(g c g-\varepsilon)_{+}=: f \in A_{+}$. Finally, we see from $\pi_{I}(g c g)=\pi_{I}(c)$ that

$$
\begin{aligned}
\left\|\pi_{I}(f)\right\| & \left.=\| \pi_{I}((g c g)-\varepsilon)_{+}\right) \|=\left(\left\|\pi_{I}(g c g)\right\|-\varepsilon\right)_{+}=\left(\left\|\pi_{I}(c)\right\|-\varepsilon\right)_{+} \\
& =\left(\left\|(x E(b) x-\varepsilon)_{+}\right\|-\varepsilon\right)_{+}=\|x E(b) x\|-\frac{1}{2}\|E(b)\|>\frac{1}{4}\|E(b)\|>0 .
\end{aligned}
$$

Hence, $f \notin I$.

The following corollary shows that in the case of commutative $A$ and discrete amenable $G$ several of the previously considered properties are equivalent.

COROLLARY 4.9. If $A$ is commutative and $G$ is a discrete amenable group that acts on $A$ by $\sigma$, then the following properties are equivalent.

(i) A separates the ideals of $A \rtimes_{\sigma} G$, i.e., $I \mapsto A \cap I$ is an injective map from $\mathcal{I}\left(A \rtimes_{\sigma} G\right)$ into $\mathcal{I}(A)$ (see [22, Definition 1.9]).

(ii) The action $\sigma: G \rightarrow \operatorname{Aut}(A)$ is residually properly outer (Definition 4.1).

(iii) The family $\mathcal{F}:=A_{+}$is filling for $A \rtimes_{\sigma} G$ (Definition 3.3).

Proof. (i) $\Rightarrow$ (ii): By [11, Theorem 4.1] (in case of unital $A$, and [1, Theorem 2] for the general—non-unital—case), the separation property implies that the adjoint action of $G$ on the Gelfand spectrum of $A$ is topologically free, which is equivalent to element-wise proper outerness by [1, Proposition 1].

This applies also to the quotients $(A / J) \rtimes_{[\sigma]_{J}, \lambda} G$, because the property (i) passes to quotients by amenability of $G$. See also [22, Theorem 1.13].

(ii) $\Rightarrow$ (iii): Since amenable $G$ are exact, the residual proper outerness of the action implies that $\mathcal{F}:=A_{+}$is filling for $A \rtimes_{\sigma, \lambda} G$ by Theorem 4.8 .

(iii) $\Rightarrow$ (i): By Remark 3.5, the subalgebra $A$ separates the ideals of $B:=A \rtimes_{\sigma, \lambda} G$ if $\mathcal{F}:=A_{+}$is filling for $B$. 
Remark 4.10. Asking $G$ to be amenable in Corollary 4.9 can be weakened to exactness of $\sigma$ and $A \rtimes_{\sigma, \lambda} G \cong A \rtimes_{\sigma} G$. One might also expect nuclearity of $A \rtimes_{\sigma, \lambda} G$ would suffice in place of amenability of $G$ (this is known at least in the unital case).

\section{Strongly purely infinite crossed products}

In this section we prove our main result (Theorem 1.1). We start with the definition of a $G$-separating action.

Definition 5.1. Suppose that $(A, G, \sigma)$ is a $C^{*}$-dynamical system with discrete group $G$. The action of $G$ on $A$ is $G$-separating if, for every $a, b \in A_{+}, c \in A, \varepsilon>0$, there exist elements $s, t \in A$ and $g, h \in G$ such that

$$
\left\|s^{*} a s-\sigma_{g}(a)\right\|<\varepsilon, \quad\left\|t^{*} b t-\sigma_{h}(b)\right\|<\varepsilon \quad \text { and } \quad\left\|s^{*} c t\right\|<\varepsilon .
$$

\section{Remarks 5.2.}

(i) Notice that Definition 3.6 and Remark 3.7 immediately imply that every action $\sigma: G \rightarrow \operatorname{Aut}(A)$ is $G$-separating if $A$ itself is strongly purely infinite: take $h=g=$ $e \in G$. If the contractions $s, t \in A$ satisfy the defining inequalities (2) of s.p.i. algebras $A$, then they also satisfy the inequalities (5).

(ii) $G$-separating actions on a locally compact space $X$ are not necessarily minimal. One can show that the mentioned example in Remark 4.6(ii) of an exact and non-minimal action of the (small) Thompson group $F$ on two parallel lines $\mathbb{R} \cup(i+\mathbb{R}) \subseteq \mathbb{C}$ is also $F$-separating.

(iii) The existence of a $G$-separating action on $A$ imposes a requirement on $A$ itself, e.g. the cases $a=b=c=p$ and $a=b=c=1$ with $\varepsilon=1 / 3$ in inequalities (5) show that $A$ cannot contain minimal non-zero projections $p \in A$ and that $1_{A}$ must be properly infinite in $A$ if $A$ is unital. Therefore, $C^{*}$-algebras that are commutative and unital cannot have any $G$-separating actions.

(iv) Further variations of the concepts that we introduce here are possible, e.g. one could start with conditions that are weaker than conditions for $G$-separating actions. Also, one could require the existence of $n \in \mathbb{N}$ such that for $a, b \in A_{+}$and $\varepsilon>0$, there is a solution $d_{1}, \ldots, d_{n} \in A$ and $g_{1}, \ldots, g_{n} \in G$ of the inequality (7) in Definition 7.1 of $n$-majorizing actions whenever $b$ is in the smallest $G$-invariant closed ideal that contains $a$. Or one could attempt to replace the filling family $\mathcal{F}:=A_{+}$by smaller filling families $\mathcal{F} \subseteq A_{+}$and require more elaborate local matrix diagonalization formulas involving also $G$-translates; cf. Definition 3.8.

Combining Theorem 4.8 with Theorem 3.13, we obtain the following result.

THEOREM 5.3. Let $G$ be a discrete group acting by $\sigma: G \rightarrow \operatorname{Aut}(A)$ on a separable or commutative $C^{*}$-algebra $A$. Suppose that the action is residually properly outer (cf. Definition 4.1) and exact (cf. Definition 4.5). Let $\mathcal{F} \subseteq A_{+}$be a filling family for $A$. Then the following are equivalent.

(i) The crossed product $A \rtimes_{\sigma, \lambda} G$ is strongly purely infinite.

(ii) The family $\mathcal{F}$ has the diagonalization property in $A \rtimes_{\sigma, \lambda} G$. 
Proof. Let $B:=A \rtimes_{\sigma, \lambda} G$. The assumptions ensure that $A_{+}$is a filling family for $B$ by Theorem 4.8. Since $\mathcal{F}$ is filling for $A, \mathcal{F}$ is also filling for $B$ by Lemma 3.4.

(i) $\Rightarrow$ (ii): If $B$ is s.p.i., then $B_{+}$has the diagonalization property (see Definition 3.9 ) in $B$; cf. [13, Lemma 5.7]. This implies that our family $\mathcal{F} \subseteq A_{+} \subseteq B_{+}$has the diagonalization property in $B$.

(ii) $\Rightarrow$ (i): Since our family $\mathcal{F} \subseteq A_{+}$is filling for $B$, and since $\mathcal{F}$ has the diagonalization property in $B$, we get that $B$ is s.p.i. by Theorem 3.13 .

Remark 5.4. Let $(A, G, \sigma)$ be a $C^{*}$-dynamical system.

(i) For each $a_{1}, a_{2} \in A_{+}, x, d_{1}, d_{2} \in A, g_{0}, g_{1}, g_{2} \in G$ and $s_{1}:=d_{1} U\left(g_{1}\right), s_{2}:=$ $\sigma_{g_{0}^{-1}}\left(d_{2}\right) U\left(g_{0}^{-1} g_{2} g_{2}\right), \quad c:=x U\left(g_{0}\right), \quad b_{1}:=a_{1}$ and $b_{2}:=\sigma_{g_{0}}\left(a_{2}\right)$, the following equalities hold:

$$
\left\|s_{j}^{*} a_{j} s_{j}-a_{j}\right\|=\left\|d_{j}^{*} b_{j} d_{j}-\sigma_{g_{j}}\left(b_{j}\right)\right\| \quad \text { and } \quad\left\|s_{1}^{*} c s_{2}\right\|=\left\|d_{1}^{*} x d_{2}\right\| .
$$

(ii) With $g_{0}=e$ in (i), the equalities reduce to

$$
\left\|s_{j}^{*} a_{j} s_{j}-a_{j}\right\|=\left\|d_{j}^{*} a_{j} d_{j}-\sigma_{g_{j}}\left(a_{j}\right)\right\| \quad \text { and } \quad\left\|s_{1}^{*} c s_{2}\right\|=\left\|d_{1}^{*} c d_{2}\right\| .
$$

Proposition 5.5. Suppose that $(A, G, \sigma)$ is a $C^{*}$-dynamical system with discrete $G$. The following properties (i)-(ii) are equivalent.

(i) The action of $G$ on $A$ is $G$-separating in the sense of Definition 5.1.

(ii) For every $a_{1}, a_{2} \in A_{+}, \quad c \in A \rtimes_{\sigma, \lambda} G$ and $\varepsilon>0$, there exist $d_{1}, d_{2} \in A$ and $g_{1}, g_{2} \in G$ such that the elements $s_{j}=d_{j} U\left(g_{j}\right)$ of $\mathrm{C}_{c}(G, A)$ satisfy, for $j=1,2$,

$$
\left\|s_{j}^{*} a_{j} s_{j}-a_{j}\right\|<\varepsilon, \quad \text { and } \quad\left\|s_{1}^{*} c s_{2}\right\|<\varepsilon .
$$

Proof. (ii) $\Rightarrow$ (i): If we take $c \in A, a_{1}:=a$ and $a_{2}:=b$ for $a, b \in A_{+}$and $\varepsilon>0$, then (ii) implies, using Remark 5.4, that there exist $d_{1}, d_{2} \in A$ and $g_{1}, g_{2} \in G$ such that $\| d_{j}^{*} a_{j} d_{j}-$ $\sigma_{g_{j}}\left(a_{j}\right) \|<\varepsilon$ and $\left\|d_{1}^{*} c d_{2}\right\|<\varepsilon$, so the inequalities (5) of Definition 5.1 are satisfied with $d_{1}, d_{2}, g_{1}, g_{2}$ in place of $s, t, g, h$.

(i) $\Rightarrow$ (ii): Define $\mathcal{C}:=\{d U(g) \mid d \in A, g \in G\}$ and $\mathcal{S}:=\mathcal{C}$. Select any $\varepsilon_{0}>0$. Clearly, the closed linear span of $\mathcal{C}$ is equal to $A \rtimes_{\sigma, \lambda} G$. If we can show that $\mathcal{F}:=A_{+}, \mathcal{C}$ and $\mathcal{S}$ satisfy the assumptions (i)-(iii) of Lemma 3.11-with $A \rtimes_{\sigma, \lambda} G$ in place of $A$-, then it follows from Lemma 3.11 that for every $a_{1}, a_{2} \in A_{+}, c \in A \rtimes_{\sigma, \lambda} G$ and $\varepsilon>0$, there exist $d_{1}, d_{2} \in A$ and $g_{1}, g_{2} \in G$ such that $s_{j}=d_{j} U\left(g_{j}\right) \in \mathcal{S}$ fulfil (6), which in turn gives (ii).

It is evident that our $\mathcal{C}$ and $\mathcal{S}$ satisfy properties (ii) and (iii) in Lemma 3.11. Since $A_{+}$ is closed under $\varepsilon$-cut-downs, property (i) in Lemma 3.11 becomes automatic if each pair $\left(a_{1}, a_{2}\right)$, with $a_{1}, a_{2} \in A_{+}$, has the matrix diagonalization property of Definition 3.8 with respect to $\mathcal{S}$ and $\mathcal{C}$ :

If $a_{1}, a_{2} \in A_{+}, c=x U\left(g_{0}\right) \in \mathcal{C}$ with $x \in A, g_{0} \in G$ and $\varepsilon>0$ are given, then we define $b_{1}:=a_{1}, b_{2}:=\sigma_{g_{0}}\left(a_{2}\right)$. (If, instead of $\varepsilon$, we are given positive $\varepsilon_{1}, \varepsilon_{2}$ and $\tau$, set $\varepsilon:=\min \left(\varepsilon_{1}, \varepsilon_{2}, \tau\right)$.) Since the action $\sigma$ is $G$-separating, we can find $d_{1}, d_{2} \in A$ and $g_{1}, g_{2} \in G$ with $\left\|d_{1}^{*} b_{1} d_{1}-\sigma_{g_{1}}\left(b_{1}\right)\right\|,\left\|d_{2}^{*} b_{2} d_{2}-\sigma_{g_{2}}\left(b_{2}\right)\right\|$ and $\left\|d_{1}^{*} x d_{2}\right\|$ all strictly below $\varepsilon$. Remark 5.4 provides elements $s_{j} \in \mathcal{C}$ satisfying (6). Thus, $\left(a_{1}, a_{2}\right)$ has the matrix diagonalization property with respect to $\mathcal{S}$ and $\mathcal{C}$. 
THEOREM 5.6. Let $(A, G, \sigma)$ be a $C^{*}$-dynamical system with discrete $G$. Suppose that $A_{+}$is a filling family for $A \rtimes_{\sigma, \lambda} G$ and that the action of $G$ on $A$ is $G$-separating. Then $A \rtimes_{\sigma, \lambda} G$ is strongly purely infinite.

Proof. By Theorem 3.13, it remains to show that $A_{+}$has the diagonalization property in $A \rtimes_{\sigma, \lambda} G$. Since $A_{+}$is closed under $\varepsilon$-cut-downs, Lemma 3.10 applies and therefore it is enough to show that each pair $\left(a_{1}, a_{2}\right)$ with $a_{1}, a_{2} \in A_{+}$has the matrix diagonalization property in $A \rtimes_{\sigma, \lambda} G$. But this follows from the $G$-separation property of the action $\sigma$ by Proposition 5.5.

Proof of Theorem 1.1. By Theorems 4.8 and 5.6, the assumptions imply that $A \rtimes_{\sigma, \lambda} G$ is strongly purely infinite.

Remark 5.7. Suppose that $(A, G, \sigma)$ is a $C^{*}$-dynamical system and that $G$ is discrete. Then a family $\mathcal{F} \subseteq A_{+} \subseteq A \rtimes_{\sigma, \lambda} G$ which is invariant under $\varepsilon$-cut-downs has the diagonalization property in $A \rtimes_{\sigma, \lambda} G$ if and only if, for every $a_{1}, a_{2} \in \mathcal{F}, c \in \mathrm{C}_{c}(G, A)$ and $\varepsilon>0$, there exist $s_{1}, s_{2} \in \mathrm{C}_{c}(G, A)$ such that, for $j=1,2$,

$$
\left\|s_{j}^{*} a_{j} s_{j}-a_{j}\right\|<\varepsilon \quad \text { and }\left\|s_{1}^{*} c s_{2}\right\|<\varepsilon .
$$

This follows from Lemma 3.10, Lemma 3.11 and the fact that $\mathrm{C}_{c}(G, A)$ is dense in $A \rtimes_{\sigma, \lambda} G$.

Remark 5.8. Notice that for an exact locally compact group $G$, the reduced group $C^{*}$-algebra $C_{\lambda}^{*}(G)$ is an exact $C^{*}$-algebra (cf. [15, p. 171]). By Theorem 3.12, the minimal $C^{*}$-tensor product $A \otimes \otimes^{\min } B$ of a s.p.i. $C^{*}$-algebra $A$ with an exact $C^{*}$-algebra $B$ is again s.p.i. Hence, if $G$ is an exact locally compact group and $\sigma(g):=\operatorname{id}_{A}$ is the trivial action on a s.p.i. $C^{*}$-algebra $A$, then $A \rtimes_{\sigma, \lambda} G \cong A \otimes^{\min } C_{\lambda}^{*}(G)$ is s.p.i.

This shows that there is room for refinements of our sufficient conditions on the actions that imply strong pure infiniteness of the reduced crossed products: here the action $\sigma$ is even not element-wise properly outer, but satisfies the $G$-separation property and is an exact action by $C^{*}$-exactness of $C_{\lambda}^{*}(G)$.

\section{The case of commutative $C^{*}$-algebras}

The case of $G$-actions on commutative $C^{*}$-algebras allows some topological interpretation. The next lemma has inspired our choice of the name $G$-separating in Definition 5.1. Notice that we do not require $\alpha_{g_{j}}\left(U_{j}\right) \subseteq \overline{U_{j}}$ in its part (ii).

Lemma 6.1. Suppose that $(A, G, \sigma)$ is a $C^{*}$-dynamical system, that $A \cong \mathrm{C}_{0}(X)$ is commutative and that the action $\sigma$ of $G$ on $\mathrm{C}_{0}(X)$ is induced by the action $\alpha$ of $G$ on $X \cong \widehat{A}$ (i.e., $\sigma_{g}(f):=f \circ \alpha_{g}^{-1}$ for $f \in A, g \in G$ ). Then the following properties are equivalent.

(i) The action of $G$ on $A$ is $G$-separating, i.e., for every $a, b \in A_{+}, c \in A$ and $\varepsilon>0$, there exist elements $d_{1}, d_{2} \in A$ and $g_{1}, g_{2} \in G$ such that

$$
\left\|d_{1}^{*} a d_{1}-\sigma_{g_{1}}(a)\right\|<\varepsilon, \quad\left\|d_{2}^{*} b d_{2}-\sigma_{g_{2}}(b)\right\|<\varepsilon \quad \text { and } \quad\left\|d_{1}^{*} c d_{2}\right\|<\varepsilon .
$$

(ii) For everyopen $U_{1}, U_{2} \subseteq X$ and compact $K_{1}, K_{2} \subseteq X$ with $K_{1} \subseteq U_{1}, K_{2} \subseteq U_{2}$, there exist $g_{1}, g_{2} \in G$ such that

$$
\alpha_{g_{1}}\left(K_{1}\right) \subseteq U_{1}, \quad \alpha_{g_{2}}\left(K_{2}\right) \subseteq U_{2}, \quad \alpha_{g_{1}}\left(K_{1}\right) \cap \alpha_{g_{2}}\left(K_{2}\right)=\emptyset .
$$


Proof. (ii) $\Rightarrow$ (i): Let $a, b \in A_{+}, c \in A$ and $\varepsilon>0$. We use assumption (ii) on

$$
\begin{gathered}
U_{1}:=a^{-1}(\varepsilon / 4, \infty)=\{x \in X \mid a(x)>\varepsilon / 4\}, \quad U_{2}:=\{x \in X \mid b(x)>\varepsilon / 4\}, \\
K_{1}:=\{x \in X \mid a(x) \geq \varepsilon / 2\}, \quad K_{2}:=\{x \in X \mid b(x) \geq \varepsilon / 2\},
\end{gathered}
$$

and find $g_{1}, g_{2} \in G$ such that

$$
\alpha_{g_{1}}\left(K_{1}\right) \subseteq U_{1}, \quad \alpha_{g_{2}}\left(K_{2}\right) \subseteq U_{2}, \quad \alpha_{g_{1}}\left(K_{1}\right) \cap \alpha_{g_{2}}\left(K_{2}\right)=\emptyset .
$$

Since $a, b \in \mathrm{C}_{0}(X)_{+}$, we have that $\overline{U_{1}} \subseteq a^{-1}[\varepsilon / 4, \infty)$ and $\overline{U_{2}} \subseteq b^{-1}[\varepsilon / 4, \infty)$ are compact subsets of $X$.

Since the compact sets $\alpha_{g_{1}}\left(K_{1}\right)$ and $\alpha_{g_{2}}\left(K_{2}\right)$ are disjoint, applications of the Tietze extension theorem give elements $e_{1}, e_{2} \in A_{+}$with $\left\|e_{j}\right\| \leq 2 / \sqrt{\varepsilon}$ and a contraction $f=f^{*} \in A$ such that

$$
e_{1}\left|\overline{U_{1}}=a^{-1 / 2}\right| \overline{U_{1}}, \quad e_{2}\left|\overline{U_{2}}=b^{-1 / 2}\right| \overline{U_{2}}, \quad f\left|\alpha_{g_{1}}\left(K_{1}\right)=-1, \quad f\right| \alpha_{g_{2}}\left(K_{2}\right)=1 .
$$

Let $f_{+}, f_{-} \in A_{+}$be the canonical decomposition $f=f_{+}-f_{-}$with $f_{+} f_{-}=0$. We define

$$
d_{1}:=e_{1}\left(\sigma_{g_{1}}(a)-\varepsilon / 2\right)_{+}^{1 / 2} f_{-} \quad \text { and } \quad d_{2}:=e_{2}\left(\sigma_{g_{2}}(b)-\varepsilon / 2\right)_{+}^{1 / 2} f_{+} .
$$

Then $d_{1}^{*} c d_{2}=0$, because $f_{+} f_{-}=0$.

Since $\left(\sigma_{g_{1}}(a)-\varepsilon / 2\right)_{+}(x) \neq 0$ implies $a\left(\alpha_{g_{1}^{-1}}(x)\right)>\varepsilon / 2$, we get $\alpha_{g_{1}^{-1}}(x) \in K_{1}$, and $x \in \alpha_{g_{1}}\left(K_{1}\right) \subseteq U_{1} \subseteq \overline{U_{1}}$. It implies that $f_{-}(x)=1$ and $e_{1}(x)=a^{-1 / 2}(x)$. We obtain that

$$
d_{1}^{*} a d_{1}=e_{1}^{2} a\left(\sigma_{g_{1}}(a)-\varepsilon / 2\right)_{+}\left(f_{-}\right)^{2}=\left(\sigma_{g_{1}}(a)-\varepsilon / 2\right)_{+} .
$$

In a similar way, we see that $d_{2}^{*} b d_{2}=\left(\sigma_{g_{2}}(b)-\varepsilon / 2\right)_{+}$.

(i) $\Rightarrow$ (ii): Let $U_{1}, U_{2} \subseteq X$ be open and $K_{1}, K_{2} \subseteq X$ be compact subsets such that $K_{1} \subseteq U_{1}$ and $K_{2} \subseteq U_{2}$. We can assume that the intersection $K_{1} \cap K_{2}$ is non-empty (if $K_{1} \cap K_{2}=\emptyset$, then property (ii) is satisfied with $g_{1}=g_{2}=e$ ). There exists an open set $W$ with a compact closure $\bar{W}$ such that

$$
K_{1} \cup K_{2} \subseteq W \subseteq \bar{W} \subseteq U_{1} \cup U_{2} .
$$

By the Tietze extension theorem, there are contractions $a, b, c \in A_{+}$such that

$$
a\left|K_{1}=1, \quad b\right| K_{2}=1, \quad c \mid \bar{W}=1,
$$$$
\operatorname{supp}(a) \subseteq U_{1} \cap W, \quad \operatorname{supp}(b) \subseteq U_{2} \cap W, \quad \operatorname{supp}(c) \subseteq U_{1} \cup U_{2} .
$$

We apply assumption (i) to $a, b, c$ and $\varepsilon:=1 / 4$, and get elements $d_{1}, d_{2} \in A$ and $g_{1}, g_{2} \in G$ such that

$$
\left\|d_{1}^{*} a d_{1}-\sigma_{g_{1}}(a)\right\|<1 / 4, \quad\left\|d_{2}^{*} b d_{2}-\sigma_{g_{2}}(b)\right\|<1 / 4, \quad\left\|d_{1}^{*} c d_{2}\right\|<1 / 4 .
$$

If $x \in \bar{W}$, then $c(x)=1$ and $\left|d_{1}(x)\left\|d_{2}(x) \mid \leq\right\| d_{1}^{*} c d_{2} \|<1 / 4\right.$. Thus,

$$
V_{1}:=\left\{x \in \bar{W}|| d_{1}(x) \mid \geq 1 / 2\right\}, \quad V_{2}:=\left\{x \in \bar{W}|| d_{2}(x) \mid \geq 1 / 2\right\}
$$

are disjoint sets. If $x \in \alpha_{g_{1}}\left(K_{1}\right)$, then $\alpha_{g_{1}^{-1}}(x) \in K_{1}$ and $\sigma_{g_{1}}(a)(x)=a\left(\alpha_{g_{1}^{-1}}(x)\right)=1$. It follows that $\left|d_{1}(x)\right|^{2} a(x) \geq 3 / 4$ and $\left|d_{1}(x)\right|>1 / 2$ (the latter because $1 \geq a(x)>0$ ). Thus, $x \in U_{1} \cap W$ (as $x \in \operatorname{supp}(a)$ ) and $x \in V_{1}$ (as $\left|d_{1}(x)\right| \geq 1 / 2$ ). Since $x$ was an arbitrary element of $\alpha_{g_{1}}\left(K_{1}\right)$, it follows that $\alpha_{g_{1}}\left(K_{1}\right) \subseteq U_{1} \cap V_{1}$. In a similar way, $\alpha_{g_{2}}\left(K_{2}\right) \subseteq$ $U_{2} \cap V_{2}$. It implies that $\alpha_{g_{1}}\left(K_{1}\right) \subseteq U_{1}, \alpha_{g_{2}}\left(K_{2}\right) \subseteq U_{2}$ and that $\alpha_{g_{1}}\left(K_{1}\right) \cap \alpha_{g_{2}}\left(K_{2}\right)=\emptyset$. 
The following condition (i) in Corollary 6.2 is satisfied if the action $\alpha$ has the residual version of the topological freeness in the sense of [1, Definition 1]; see e.g. the essential freeness defined in [22, Definition 1.17] (inspired by [20, Definition 4.8]) when $G$ is countable.

COROLlary 6.2. Let $G$ be a discrete group and $\alpha: G \rightarrow \operatorname{Homeo}(X)$ an action of $G$ on a locally compact Hausdorff space X. Suppose that:

(i) for every closed $G$-invariant subset $Y$ of $X$ and every $e \neq g \in G$, the set $\{y \in Y$ : $\left.\alpha_{g}(y)=y\right\}$ has empty interior;

(ii) the action $\sigma: G \rightarrow \operatorname{Aut}\left(\mathrm{C}_{0}(X)\right)$, given by $\sigma_{g}(f):=f \circ\left(\alpha_{g}\right)^{-1}$, is exact on the $C^{*}$-algebra $\mathrm{C}_{0}(X) ;$ and

(iii) the action $\alpha$ is $G$-separating, i.e., by Lemma 6.1, for every $U_{1}, U_{2} \subseteq X$ open and $K_{1}, K_{2} \subseteq X$ compact such that $K_{1} \subseteq U_{1}, K_{2} \subseteq U_{2}$, there exist $g_{1}, g_{2} \in G$ such that

$$
\alpha_{g_{1}}\left(K_{1}\right) \subseteq U_{1}, \quad \alpha_{g_{2}}\left(K_{2}\right) \subseteq U_{2}, \quad \alpha_{g_{1}}\left(K_{1}\right) \cap \alpha_{g_{2}}\left(K_{2}\right)=\emptyset .
$$

Then $\mathrm{C}_{0}(X) \rtimes_{\sigma, \lambda} G$ is a strongly purely infinite $C^{*}$-algebra.

Proof. Let $A:=\mathrm{C}_{0}(X)$. It is easy to see that property (i) implies that the action on any quotient $A / I$ by a $G$-invariant closed ideal $I \neq A$ is element-wise properly outer. Now Theorem 1.1 (proved in §5) applies to $A \rtimes_{\sigma, \lambda} G$.

\section{Strong boundary actions versus G-separating actions}

Here we prove Theorem 1.2. We start with the definitions of $n$-majorizing and $n$-covering actions.

Definition 7.1. Let $n \in \mathbb{N}$ and $A$ be a non-zero $C^{*}$-algebra, that is not isomorphic to a subalgebra of $M_{n+1}(\mathbb{C})$ if $A$ is unital. An action $\sigma: G \rightarrow \operatorname{Aut}(A)$ will be called an $n$-majorizing action of $G$ on $A$ if, for every non-zero $a \in A_{+}$, every non-invertible $b \in A_{+}$ and every $\varepsilon>0$, there exist $d_{1}, \ldots, d_{n} \in A$ and $g_{1}, \ldots, g_{n} \in G$ such that

$$
\left\|\sum_{j=1}^{n} d_{j}^{*} \sigma_{g_{j}}(a) d_{j}-b\right\|<\varepsilon .
$$

Definition 7.2. Let $n \in \mathbb{N}, n \geq 2 \dagger$. Suppose that $A$ is a unital $C^{*}$-algebra, that is not isomorphic to a *-subalgebra of $M_{n}(\mathbb{C})$. An action $\sigma$ of $G$ on $A$ is an n-covering action if, for every non-zero $a \in A^{+}$and every $\varepsilon>0$, there exist $d_{1}, \ldots, d_{n} \in A$ and $g_{1}, g_{2}, \ldots, g_{n} \in G$ such that

$$
\left\|\sum_{j=1}^{n} d_{j}^{*} \sigma_{g_{j}}(a) d_{j}-1\right\|<\varepsilon .
$$

The following lemma denies the existence of non-zero 'socles' in $C^{*}$-algebras $A$ that admit $n$-majorizing or $n$-covering actions considered in Definitions 7.1 and 7.2.

LEMMA 7.3. Let $(A, G, \sigma)$ be a $C^{*}$-dynamical system.

Consider the following properties $(\alpha)$ or $(\beta)$ of $(A, G, \sigma)$ depending on $n \in \mathbb{N}$.

$\dagger$ If $n=1$, then property (8) holds if and only if $A$ is a unital simple purely infinite $C^{*}$-algebra. 
( $\alpha)$ There is $n \geq 1$ such that, for each non-zero $a \in A_{+}$, non-invertible $b \in A_{+}$and $\varepsilon>0$, there exist $d_{1}, \ldots, d_{n} \in A$ and $g_{1}, \ldots, g_{n} \in G$ that satisfy the inequality (7) in Definition 7.1.

( $\beta) \quad A$ is unital, and there is $n \geq 2$ such that, for each non-zero $a \in A_{+}$and $\varepsilon>0$, there exist $d_{1}, \ldots, d_{n} \in A$ and $g_{1}, \ldots, g_{n} \in G$ that satisfy the inequality (8) in Definition 7.2.

If $A$ is unital and $(A, G, \sigma)$ satisfies $(\alpha)$, then it satisfies $(\beta)$ with $n$ replaced by $n+1$ and, if $(A, G, \sigma)$ satisfies $(\beta)$, then it satisfies $(\alpha)$-with the same $n \in \mathbb{N}$. If $(A, G, \sigma)$ satisfies $(\alpha) \operatorname{or}(\beta)$, then the algebra $A$ is $G$-simple, i.e., $A$ and $\{0\}$ are the only $G$-invariant closed ideals of $A$.

If A contains a projection $p \neq 0$ with $p A p=\mathbb{C} \cdot p$, then $A$ is a $C^{*}$-subalgebra of $M_{n+1}$ (respectively of $\left.M_{n}\right)$ if $(A, G, \sigma)$ has property $(\alpha)$ (respectively has property $(\beta)$ ).

The shift action $\sigma$ of the cyclic group $\mathbb{Z}_{n+1}:=\mathbb{Z} /(n+1) \mathbb{Z}$ on $A:=\mathrm{C}\left(\mathbb{Z}_{n+1}\right)$ satisfies ( $\alpha$ ) for $n \in \mathbb{N}$ and is element-wise properly outer.

Proof. If $A$ is unital and $\sigma$ has property $(\alpha)$, then let $b:=1-\left(\|a\|^{-1} a\right)^{3}$, and find $d_{1}, \ldots, d_{n}$ and $g_{1}, \ldots, g_{n}$ that satisfy the inequality (7). If we let $g_{n+1}:=e$ and $d_{n+1}:=\|a\|^{-3 / 2} a$, then $a$ and $g_{1}, \ldots, g_{n}, g_{n+1}$ satisfy (8). It shows that actions on unital $A$ that satisfy property $(\alpha)$ are also actions that satisfy $(\beta)$ with $n+1$ in place of $n$. If $(A, G, \sigma)$ satisfies $(\beta)$ and non-zero elements $a, b \in A_{+}$are given with $\|b\|=1$, then $d_{1} b^{1 / 2}, \ldots, d_{n} b^{1 / 2}$ and $g_{1}, \ldots, g_{n}$ is a solution of the inequality (7) in Definition 7.1 if $d_{1}, \ldots, d_{n}$ and $g_{1}, \ldots, g_{n}$ satisfy the inequality (8) of Definition 7.2.

The properties $(\alpha)$ and $(\beta)$ imply that $\{0\}$ and $A$ are the only $G$-invariant closed ideals of $A$ : if $A$ is non-unital in case $(\alpha)$, then the approximation, as expressed by the inequalities (7), shows that for each non-zero $a \in A_{+}$the smallest closed $G$-invariant ideal of $A$ containing $a$ contains each $b \in A_{+}$. If $A$ is unital and the action has property $(\beta)$, then each $G$-invariant closed ideal of $A$ contains 1 . If $A$ is unital and the $C^{*}$-dynamical system $(A, G, \sigma)$ satisfies property $(\alpha)$, then it satisfies property $(\beta)$ with $n$ replaced by $n+1$. Thus, again, $A$ and $\{0\}$ are the only closed $G$-invariant ideals.

From now on, we suppose that there exists a projection $0 \neq p \in A_{+}$with $p A p=\mathbb{C} p$. We call those projections 'minimal', even if minimal non-zero projections of a $C^{*}$-algebra $A$ do not have the property $p A p=\mathbb{C} p$ in general, e.g. the unit of the Jiang-Su algebra $\mathcal{Z}$ is a minimal projection. We show that this assumption of the existence of such $p \in A$, together with the assumption that $\sigma$ satisfies $(\alpha)$, implies that $A$ is unital. Thus, $A$ satisfies $(\beta)$ with $n+1$ in place of $n$. Then we derive that property $(\beta)$ and the existence of such $p \in A$ imply that $A$ is a $C^{*}$-subalgebra of $M_{n}$.

It is obvious that the ideal $\operatorname{socle}(A)$ generated by those 'minimal' projections is (universally) invariant under all automorphisms of $A$, i.e., $\alpha(\operatorname{socle}(A))=\operatorname{socle}(A)$ for all $\alpha \in \operatorname{Aut}(A)$. This happens also for the closure $J$ of socle $(A)$. Thus, $J$ must be $G$-invariant. Since $A$ is $G$-simple by $(\alpha)$ and $\operatorname{socle}(A) \neq \emptyset$, it follows that $J=A$.

It is not difficult to see that $J$ is isomorphic to the $c_{0}$-direct sum of a family of algebras $\mathcal{K}\left(\mathcal{H}_{\tau}\right)$ of compact operators on suitable Hilbert spaces $\mathcal{H}_{\tau}$, and that $p$ is a rank-one projection on some $\mathcal{H}_{\tau_{0}}$. Let $\mathcal{H}$ denote the Hilbert space sum of the Hilbert spaces $\mathcal{H}_{\tau}$. Then $A$ becomes isomorphic to a non-degenerate $C^{*}$-subalgebra of the algebra of compact 
operators $\mathcal{K}(\mathcal{H})$ on $\mathcal{H}$, in a way that each minimal non-zero projection $p \in A$ becomes a rank-one projection on $\mathcal{H}$. This happens also for all $\sigma_{g}(p)$. Recall that every projection in $A \subseteq \mathcal{K}(\mathcal{H})$ has finite rank in $\mathcal{L}(\mathcal{H})$. Since $A$ is a $C^{*}$-subalgebra of $\mathcal{K}(\mathcal{H}), A$ is in particular an approximately finite-dimensional $C^{*}$-algebra, and-therefore-contains an approximate unit $\left(q_{\tau}\right)$ consisting of an upward-directed net of projections of finite rank in $\mathcal{H}$.

We show that $A$ must be unital using that $(A, G, \sigma)$ satisfies $(\alpha)$ by an indirect proof: suppose that $A$ is not unital; then none of the projections $\left(q_{\tau}\right)$ are invertible in $A$. Therefore, we can take $b:=q_{\tau}, a:=p$ and $\varepsilon=1 / 2$ in (7). It follows that each $q_{\tau}$ has linear rank $\leq n$. This implies that the approximate unit $\left(q_{\tau}\right)$ must be constant, $q_{\tau}=e$, for all $\tau \geq \tau_{1}$ with suitable $\tau_{1}$. Then $e \in A$ is necessarily the unit element of $A$, in contradiction to our assumption that $A$ is not unital.

It follows that $A$ must be unital, and-as above observed-the action $\sigma$ satisfies property $(\beta)$ with $n$ replaced by $n+1$.

If $A$ is unital and $(A, G, \sigma)$ satisfies property $(\beta)$, then we take $a:=p$ and $\varepsilon:=1 / 2$ in inequalities (8). It shows that the rank of $1_{A}$ in its representation is $\leq n$. Thus, $A$ is a $C^{*}$-subalgebra of $M_{n}$ in case $(\beta)$.

The crossed product $\mathrm{C}\left(\mathbb{Z}_{n+1}\right) \rtimes \mathbb{Z}_{n+1}$, where $\mathbb{Z}_{n+1}=\mathbb{Z} /(n+1) \mathbb{Z}$, is naturally isomorphic to $M_{n+1}$. Hence, by Corollary 4.9 , the action of $\mathbb{Z}_{n+1}$ on $\mathrm{C}\left(\mathbb{Z}_{n+1}\right)$ is element-wise properly outer. If $a \in A_{+}:=\mathrm{C}\left(\mathbb{Z}_{n+1}\right)_{+}$is non-zero and $b \in A_{+}$is not invertible, then there are non-zero minimal projections $p, q \in A_{+}$and $\delta>0$ such that $a \geq \delta p$ and $b \leq\|b\| \cdot(1-q)$. Select $g_{1}, \ldots, g_{n} \in \mathbb{Z}_{n+1}$ with $\sum_{j=1}^{n} \sigma_{g_{j}}(p)=1-q$. It implies that $\delta^{-1} \sum_{j=1}^{n} \sigma_{g_{j}}(a) \geq 1-q$. Thus, there exists $T \in(1-q) A_{+}(1-q)$ with $T\left(\sum_{j=1}^{n} \sigma_{g_{j}}(a)\right) T=1-q$. Then $a, b, d_{j}:=T b^{1 / 2}, j=1, \ldots, n$ and $g_{1}, \ldots, g_{n}$ satisfy the inequality (7) for each $\varepsilon>0$.

Remark 7.4. Let $B$ be a non-zero simple $C^{*}$-algebra. In preparation for the proof of Theorem 1.2, we display here a number of properties equivalent to strong pure infiniteness of $B$.

(i) $B$ is strongly purely infinite.

(ii) Each non-zero element of $B_{+}$is properly infinite in the sense of [12].

(iii, $n$ ) There exists $n \in \mathbb{N}$ such that, for each non-zero element $a, b \in B_{+}$and $\varepsilon>0$, there exists $n$ elements $d_{1}, \ldots, d_{n} \in B$ with

$$
\left\|d_{1}^{*} a d_{1}+\cdots+d_{n}^{*} a d_{n}-b\right\|<\varepsilon,
$$

and $B$ is not isomorphic to $M_{k}$ for any $k \leq n$.

(iv) $\quad B$ is locally purely infinite in the sense of [3, Definition 1.3], i.e., each non-zero hereditary $C^{*}$-subalgebra of $B$ contains a non-zero stable $C^{*}$-subalgebra.

(v) $B$ is purely infinite in the sense of Cuntz [5, p. 186], i.e., each non-zero hereditary $C^{*}$-subalgebra contains an infinite projection.

Proof. Property (ii) implies (i) by [3, Theorem 5.8] and (i) implies (ii) by [13, Proposition 5.4]. Property (iii, $n=1$ ) is equivalent to (ii) by [12, Theorem 4.16]. The properties (iii, $n=1$ ), (iv) and (v) are equivalent by [3, Proposition 3.1]. 
(iii) $\Rightarrow$ (ii): Suppose that $B$ is elementary, i.e., that $B \cong \mathcal{K}(\mathcal{H})$ for some Hilbert space $\mathcal{H}$. By (iii), applied on some rank-one projection $a:=p \in B_{+}, b \in B_{+}$and $\varepsilon=1 / 2$, it follows that each element of $b \in B_{+}$has rank $\leq n$. Thus, $\mathcal{H}$ has dimension $k \leq n$, and $B \cong M_{k}$. But the latter case was excluded in (iii, $n$ ). Therefore, $B$ is non-elementary and hence has the global Glimm halving property in the sense of [3, Definition 2.6]. This is easy to see for non-elementary simple $B$ (or use Glimm halving [19, Lemma 6.7.1]). Since $B$ is simple, property (iii, $n$ ) ensures that $B$ satisfies property (i) of [3, Definition 1.2] of $\operatorname{pi}(n)$. Therefore, [3, Proposition 4.14] says that $B$ is pi(1). Since $B$ is simple and $B \neq \mathbb{C}$, there are no non-zero characters on $B$. In particular, pi(1) ensures that $B$ is purely infinite in the sense of [12, Definition 4.1]. By [12, Theorem 4.1] we obtain property (ii).

Proof of Theorem 1.2. By Lemma 7.3, $A$ is $G$-simple. Thus, the action $\sigma$ is automatically exact by Definition 4.5. Since $\sigma$ is element-wise properly outer by assumption, it is now also residually properly outer, and Theorem 4.8 applies. It says that $\mathcal{F}:=A_{+}$is a filling family in $A \rtimes_{\sigma, \lambda} G$. In particular, $A$ separates the ideals of the reduced crossed product. It shows that $B:=A \rtimes_{\sigma, \lambda} G$ is simple.

Take any $b \in B_{+}$with $\|b\|=1$. (Unfortunately, it not always the case that there exists $z \in B_{+}$such that $z^{*} z=b$ and $z z^{*} \in A$; if that were true then $A=B=A \rtimes_{\sigma, \lambda} G$.) Using that $A_{+}$is filling for $B$, we can find non-zero $z \in B$ such that $z^{*} z \leq b$ and $z z^{*} \in A$ : to see this, set

$$
a^{\prime}:=(3 b-2)_{+}, \quad b^{\prime}:=(3 b-1)_{+}-a^{\prime}, \quad c^{\prime}:=3 b-a^{\prime}-b^{\prime} .
$$

By functional calculus, we have $a^{\prime}, b^{\prime}, c^{\prime} \in B$ with $0 \leq a^{\prime} \leq b^{\prime} \leq c^{\prime} \leq 1, a^{\prime} b^{\prime}=a^{\prime} \neq 0$ and $b^{\prime} c^{\prime}=b$. For example, since $c^{\prime}=3 b-(3 b-1)_{+}$, we have $c^{\prime} \leq 1$ from the inequality $3 t-$ $\max (3 t-1,0) \leq 1, t \in \mathbb{R}$. By property (i) of Definition 3.3, there exist $z_{1}, z_{2}, \ldots, z_{n}$ $\in B$ and $d \in B$ with $z_{j}\left(z_{j}\right)^{*} \in A_{+}$such that $e c^{\prime}=e$ and $d^{*} e d=a^{\prime}$ for $e:=z_{1}^{*} z_{1}+\cdots+$ $z_{n}^{*} z_{n}$. Since $d^{*} e d=a^{\prime} \neq 0$, we know that $e$ is non-zero and hence so is $z:=z_{j}$ for suitable $j$. We conclude that $z^{*} z \leq e=c^{\prime} e c^{\prime} \leq\left\|c^{\prime}\right\|\|e\| c^{\prime} \leq\left\|c^{\prime}\right\|\|e\| 3 b$. Rescaling $z$ gives $z^{*} z \leq b$ (and $z z^{*} \in A$ ).

Take $\delta \in\left(0,\|z\|^{2} / 2\right)$. Then $0 \neq z\left(z^{*} z-\delta\right)_{+} z^{*}=\varphi\left(z z^{*}\right) \in A_{+}$for some suitable $\varphi \in \mathrm{C}_{c}(0, \infty]_{+}$.

We consider three cases: (i) the action is $n$-majorizing and $A$ is non-unital, (ii) the action is $n$-majorizing and $A$ is unital and (iii) the action is $n$-covering and $A$ is unital.

Since $G$ is discrete, $A$ is a non-degenerate $C^{*}$-subalgebra of $B$. In particular, $A_{+}$ contains an approximate unit $\left(e_{\nu}\right)$ of positive contractions in $A_{+}$for $B$, which we will use for case (i). In cases (ii) and (iii), let $e_{\nu}:=1$, where 1 is the unit of $A$. Define $m:=n+1$ for case (ii) and $m:=n$ for cases (i) and (iii). By each of the Definitions 7.1 and 7.2 (and using Lemma 7.3 to get property $(\beta)$ in case (ii) with $n$ replaced by $m$ ), for each $\varepsilon>0$ and $e_{\nu} \in A_{+}$there are $d_{1}, \ldots, d_{m} \in A$ and $g_{1}, \ldots, g_{m} \in G$ such that $\left\|e_{v}-\sum_{j=1}^{m} f_{j}^{*}\left(z\left(z^{*} z-\delta\right)_{+} z^{*}\right) f_{j}\right\|<\varepsilon$ for $f_{j}:=U\left(g_{j}^{-1}\right) d_{j}$ in $B$.

By Remark 2.1(ii), there exists a contraction $d_{0} \in B$ with $d_{0}^{*} b d_{0}=\left(z^{*} z-\delta\right)_{+}$. Then the elements $y_{j}:=d_{0} z^{*} f_{j} \in B$ satisfy $\left\|e_{\nu}-\sum_{j=1}^{m} y_{j}^{*} b y_{j}\right\|<\varepsilon$. Since $a^{1 / 2} e_{\nu} a^{1 / 2}$ converges to $a \in B_{+}$, we get that $B$ has the following property.

For any two non-zero elements $b, a \in B_{+}$and $\varepsilon>0$, there exist $m$ elements $d_{1}, \ldots, d_{m} \in B$ such that $\left\|a-\sum_{j=1}^{m} d_{j}^{*} b d_{j}\right\|<\varepsilon$. A simple $C^{*}$-algebra $B$ with this 
property is strongly purely infinite by Remark 7.4 if $B$ is not isomorphic to $M_{k}$ for some $k \leq m$. The case that $B$ is isomorphic to a $C^{*}$-subalgebra of $M_{m}$ has been excluded by the definitions. For case (i), we know that $A$ is non-unital and hence $B$ is non-unital. For cases (ii) and (iii), we know that $A$ is not isomorphic to a $C^{*}$-subalgebra of $M_{m}$ and hence $B$ is not isomorphic to a $C^{*}$-subalgebra of $M_{m}$.

LEMMA 7.5. The following are equivalent for $C^{*}$-algebras $B$.

(i) $B$ does not contain a non-zero projection $p \in B_{+}$with $p B p=\mathbb{C} \cdot p$.

(ii) For every non-zero hereditary $C^{*}$-subalgebra $D$ of $B$, each maximal commutative $C^{*}$-subalgebra $C$ of $D$ has perfect Gelfand spectrum $\widehat{C}$, i.e., $\widehat{C}$ does not contain an isolated point.

(iii) For every $a_{1}, a_{2} \in B_{+} \backslash\{0\}$ and $c \in B$, there are $b_{1}, b_{2} \in B_{+} \backslash\{0\}$ with $b_{1} c b_{2}=0$, and $b_{j} \leq a_{j}(j=1,2)$.

Proof. (iii) $\Rightarrow$ (i): Let $p^{*}=p=p^{2} \in B \backslash\{0\}$ and put $a_{k}:=c:=p$ for $k=1,2$. Then there are non-zero $b_{1}, b_{2} \in(p B p)_{+}$with $b_{1} b_{2}=0$. Thus, $p B p \neq \mathbb{C} \cdot p$.

(i) $\Rightarrow$ (ii): Let $D \neq\{0\}$ be a hereditary $C^{*}$-subalgebra of $B$ and $C$ a maximal commutative $C^{*}$-subalgebra of $D$. Suppose that $\widehat{C}$ is not perfect. Then $\widehat{C}$ contains an isolated point $\chi$. The point $\chi$ corresponds to a projection $0 \neq p \in C_{+} \subseteq D_{+}$with $p B p=\mathbb{C} \cdot p$; see [21, Lemma 7.1.4].

(ii) $\Rightarrow$ (iii): It is easy to see that a commutative $C^{*}$-algebra $C$ with a perfect spectrum $\widehat{C}$ contains non-zero contractions $e_{1}, e_{2} \in C_{+}$with $e_{1} e_{2}=0$, because the locally compact Hausdorff space $\widehat{C}$ must in particular contain two different points $(\neq \infty)$.

Given $c \in B$ and non-zero $a_{1}, a_{2} \in B_{+}$, let $d_{j}:=\left(a_{j}-\left\|a_{j}\right\| / 2\right)_{+}$and $x:=d_{1}^{1 / 2} c d_{2}^{1 / 2}$. Notice that $0 \neq d_{j} \in B_{+}$, and that $0 \neq d_{j}^{1 / 2} y_{j} d_{j}^{1 / 2} \leq a_{j}$ for all non-zero contractions $0 \leq y_{j} \in \overline{d_{j} B d_{j}}$. If $x=0$, then take $b_{j}:=d_{j}$. If $x \neq 0$, consider the hereditary $C^{*}$-subalgebra $D:=\overline{x^{*} B x}=\overline{x^{*} x B x^{*} x}$ that is generated by $x^{*} x$ and is contained in $\overline{d_{2}^{*} B d_{2}}$. Let $C$ be a maximal commutative $C^{*}$-subalgebra of $D$ with $x^{*} x \in C$. Then $C$ contains non-zero contractions $e_{1}, e_{2} \in D_{+}$with $e_{1} e_{2}=0=e_{1}\left(x^{*} x\right)^{1 / 2} e_{2}$.

It is well known (and easy to see) that the polar decomposition $x=v\left(x^{*} x\right)^{1 / 2}$ in $B^{* *}$ has the property that $v D v^{*}=\overline{x B x^{*}} \subseteq \overline{d_{1}^{*} B d_{1}}$. Thus, $f:=v e_{1} v^{*} \in \overline{x B x^{*}}$ and has the property $f x e_{2}=v e_{1}\left(x^{*} x\right)^{1 / 2} e_{2}=0$. It follows that $b_{1}:=d_{1}^{1 / 2} f d_{1}^{1 / 2}$ and $b_{2}:=d_{2}^{1 / 2} e_{2} d_{2}^{1 / 2}$ satisfy $b_{1} c b_{2}=d_{1}^{1 / 2} f x e_{2} d_{2}^{1 / 2}=0$ and $0 \neq b_{j} \leq a_{j}$.

Proposition 7.6. Let $(A, G, \sigma)$ be a $C^{*}$-dynamical system with non-zero non-unital $A$. If the action $\sigma$ of $G$ on $A$ is an 1-majorizing action in the sense of Definition 7.1, then $A$ is $G$-simple and $\sigma$ is $G$-separating for $A$.

Proof. The algebra $A$ is $G$-simple and $A$ does not contain a projection $p \neq 0$ with $p A p=\mathbb{C} \cdot p$ by Lemma 7.3. To show that $\sigma$ is $G$-separating, let $a_{1}, a_{2} \in A_{+} \backslash\{0\}, c \in A$ and $\varepsilon>0$. By Lemma 7.5, there exist $b_{1}, b_{2} \in A_{+} \backslash\{0\}$ with $b_{1} c b_{2}=0$ and $b_{j} \leq a_{j}$ ( $j=1,2$ ). Using (twice) that the action is 1-majorizing, we find $e_{j} \in A, h_{j} \in G$ for $j=1,2$ such that $\left\|e_{j}^{*} \sigma_{h_{j}}\left(b_{j} a_{j} b_{j}\right) e_{j}-a_{j}\right\|<\varepsilon$. With $g_{j}:=h_{j}^{-1}$ and $d_{j}:=b_{j} \sigma_{g_{j}}\left(e_{j}\right)$, we get $\left\|d_{j}^{*} a_{j} d_{j}-\sigma_{g_{j}}\left(a_{j}\right)\right\|<\varepsilon$ and $d_{1}^{*} c d_{2}=0$, i.e., $\sigma$ is $G$-separating. 
Remark 7.7. Suppose that $(A, G, \sigma)$ is a $C^{*}$-dynamical system, $A$ is unital and commutative and $G$ is discrete. Then the following properties (i)-(iv) of the action $\sigma$ are equivalent.

(i) The action is 2-covering in the sense of Definition 7.2.

(ii) The corresponding (adjoint) action $\widehat{\sigma}$ on $\widehat{A}$ is a strong boundary action in the sense of Definition 3.1.

(iii) The action is 1-majorizing in the sense of Definition 7.1.

(iv) The action is 2-filling in the sense of Definition 3.2, and $A$ is not isomorphic to a subalgebra of $M_{2}(\mathbb{C})$.

We do not know if, also for non-commutative and unital $A$, every 2-covering action is a 1-majorizing action or a 2-filling action.

Proof. We show more general implications, except for (i) $\Rightarrow$ (ii). In particular, we show that an action $\sigma$ on a unital abelian $C^{*}$-algebra $A$ is $n$-filling if and only if it is $n$-covering provided that the (linear) dimension of $A$ is greater than $n$.

(i) $\Rightarrow$ (iv) (for $A$ unital, commutative, any $n$ ):

Suppose that $A \cong \mathrm{C}(X)$ and take any $n \geq 2$. Let $\alpha$ denote the action of $G$ on $X$ inducing $\sigma$, i.e., $\sigma_{g}(f)=f \circ \alpha_{g}^{-1}$ for $f \in A$ and $g \in G$. Since the action $\alpha$ is minimal by Lemma 7.3, Remark [10, Remark 0.4] shows that it suffices to prove that for each nonempty open subset $U$ of $X$, there exist $g_{1}, \ldots, g_{n} \in G$ such that $\alpha_{g_{1}}(U) \cup \cdots \cup \alpha_{g_{n}}(U)=$ $X$. Let such $U$ be given. Select non-zero $a \in A_{+}$with support contained in $U$. By (i), there exist $g_{1}, \ldots, g_{n} \in G$ and $d_{1}, \ldots, d_{n} \in A$ such that $\sum_{j=1}^{n} d_{j}^{*} \sigma_{g_{j}}(a) d_{j} \geq \frac{1}{2}$. In particular, for each $x \in X, \sigma_{g_{j}}(a)(x)$ is non-zero for some $j$, so $x \in \alpha_{g_{i}}(U)$.

(iv) $\Rightarrow$ (i) (for $A$ unital, commutative/non-commutative, any $n$ ):

Suppose that $A$ is unital and take any $n \geq 2$. Let $0 \neq a \in A_{+}$. Using (iv), there are $g_{1}, \ldots, g_{n} \in G$ and $\delta>0$ such that $D:=\sum_{j=1}^{n} \sigma_{g_{j}}(a) \geq \delta 1$, and $A$ is not isomorphic to a $C^{*}$-subalgebra of $M_{n}$. Thus, $D$ is invertible in $A$ and $\sum_{j=1}^{n} d_{j}^{*} \sigma_{g_{j}}(a) d_{j}=1$ for $d_{j}:=D^{-1 / 2}$ in $A$.

(iii) $\Rightarrow$ (i) (for $A$ unital, commutative/non-commutative, any $n$ ):

Each $n$-majorizing action on unital $A$ is an $(n+1)$-covering action by Lemma 7.3.

(i) $\Rightarrow$ (ii) (for $A$ unital, commutative, one $n$ ):

Suppose that $A \cong \mathrm{C}(X)$ and let $\alpha$ denote the action of $G$ on $X$ inducing $\sigma$. The equivalence of (i) and (iv) shows that for given $0 \neq a \in A_{+}$, there exist $g \in G$ and $\delta>0$ such that $a+\sigma_{g}(a) \geq \delta 1$.

Let $U \subseteq X$ be open and non-empty. There is $0 \neq a \in \mathrm{C}(X)+$ with support $a^{-1}(0, \infty) \subseteq$ $U$. Choose $h \in G$ and $\delta>0$ with $a+\sigma_{h}(a) \geq \delta 1$. Since $a$ is a positive function, this implies that $\sigma_{h}(a)(x)>0$ for all points $x \in X \backslash U$. Thus, $\alpha_{g}(x) \in U$ for all $x \in X \backslash U$ and $g:=h^{-1}$, i.e., there exists $g \in G$ with $\alpha_{g}(X \backslash U) \subseteq U$.

Given non-empty open subsets $U$ and $V$ of $X$, we let $W:=U \cap V$ if $U \cap V \neq \emptyset$. Then $g \in G$ with $\alpha_{g}(X \backslash W) \subseteq W$ satisfies $\alpha_{g}(X \backslash U) \subseteq V$. If $U \cap V=\emptyset$, then we find $g, h \in G$ with $\alpha_{g}(X \backslash U) \subseteq U \subseteq X \backslash V$ and $\alpha_{h}(X \backslash V) \subseteq V$. Then $\alpha_{h g}(X \backslash U) \subseteq V$.

The space $X$ contains more than two points because $A$ is not isomorphic to a $C^{*}$-subalgebra of $M_{2}(\mathbb{C})$. Thus, $(X, G, \alpha)$ satisfies the conditions of Definition 3.1 of a strong boundary action. 
(ii) $\Rightarrow$ (iii) (for $A$ unital/non-unital, commutative, one $n$ ):

We show (iii) using possibly less than (ii): let $X$ be a locally compact space that is not necessarily compact and contains more than two points. Let $\alpha$ be an action of $G$ on $X$ with the property that, for every compact subset $K \subseteq X$ with $K \neq X$ and each non-empty open subset $U \subseteq X$, there exists $g \in G$ with $\alpha_{g}(K) \subseteq U$. Then the adjoint action $\sigma$ of $\alpha$ on $A:=\mathrm{C}_{0}(X)$ is a 1-majorizing action of $G$ on $A$.

Indeed: let $0 \neq a \in A_{+}, b \in A_{+}$non-invertible and $\varepsilon>0$. Put $\delta:=\varepsilon / 3$. Then, considered as functions on $X$, they have the property that $U:=a^{-1}(\|a\| / 2, \infty)$ is nonempty and open and $K:=b^{-1}[\delta, \infty)$ is compact. Find $h \in G$ with $\alpha_{h}(K) \subseteq U$. Then $x \in K \Leftrightarrow b(x) \geq \delta$ implies that for $g:=h^{-1}$, we get $\sigma_{g}(a)(x)=a\left(\alpha_{h}(x)\right)>\|a\| / 2$. It follows that $\left\|d^{*} \sigma_{g}(a) d-b\right\|<\varepsilon$ with $d \in A_{+}$given by $d(x):=\sigma_{g}(a)(x)^{-1 / 2}(b(x)-$ $2 \delta)_{+}^{1 / 2}$ for $x \in K$ and $d(x):=0$ for $x \in X \backslash K$.

Remarks 7.8. (i) Let $\alpha$ be an action of a discrete group $G$ on a locally compact Hausdorff space $X$ with more than two points, and $\sigma$ the induced action on $A:=\mathrm{C}_{0}(X)$. By Remark 7.7, the following properties are equivalent for $X$ compact.

(1) The action $\alpha$ is a strong boundary action (Definition 3.1) in the sense of [17]: for each pair of non-empty open subsets $U$ and $V$ of $X$, there exists $g \in G$ with $U \cup \alpha_{g}(V)=X$.

(2) For any compact set $K \neq X$ and any open set $U \neq \emptyset$, there exists $t \in G$ such that $\alpha_{t}(K) \subseteq U$

(3) For every non-zero $a \in A_{+}$, every non-invertible $b \in A_{+}$and every $\varepsilon>0$, there exist $d \in A$ and $g \in G$ such that $\left\|d^{*} \sigma_{g}(a) d-b\right\|<\varepsilon$.

Clearly, this cannot be the case if $X$ is locally compact but is not compact. In general (i.e., when $X$ is compact or non-compact), we know that (1) $\Rightarrow(2) \Rightarrow(3)$. Properties (2) and (3) are both candidates for a generalized notion of a strong boundary action; however, only (3) applies when $A$ is non-commutative.

(ii) The notion of a strong boundary action (Definition 3.1) is defined on compact Hausdorff spaces with more than two points. In view of Remarks 7.8(i) and 7.7, we call the 1-majorizing actions on not-necessarily unital or commutative $C^{*}$-algebras also strong boundary actions.

(iii) Suppose that a discrete group $G$ acts by a topologically free action $\alpha$ on a compact Hausdorff space $X$, and that $X$ contains more than two points. It was shown in [17, Theorem 5] that the crossed product $\mathrm{C}(X) \rtimes_{\sigma, \lambda} G$ is purely infinite provided that the action - in addition - is a strong boundary action. Since topological freeness implies that $\sigma$ is element-wise properly outer (by [1, Proposition 1]), we conclude that, with the terminology of Remark 7.8(ii), [17, Theorem 5] is a special case of Theorem 1.2.

(iv) Let $\alpha$ be an action on a non-compact locally compact Hausdorff space $X$ with more than two points and $\sigma$ the induced action. It was shown in Proposition 7.6 that $\sigma$ is $G$-separating if $\sigma$ is a strong boundary (i.e., 1-majorizing) action. A simpler argument applies if we assume that for any compact set $K \neq X$ and any non-empty open set $U \subseteq X$, there exists $g \in G$ such that $\alpha_{g}(K) \subseteq U$.

Proof of (iv). Since any finite subset $M$ of $X$ is compact, it can be moved by suitable $\alpha_{g}$ into any non-empty open subset $U$ of $X$. In particular, $X$ is perfect and each non-empty 
open set $V \subseteq X$ contains at least two non-empty open disjoint subsets $V_{1}$ and $V_{2}$. Let $K_{1} \subseteq U_{1}$ and $K_{2} \subseteq U_{2}$ with $K_{j}$ compact (hence $K_{j} \neq X$ ) and $U_{j}$ open. If $U_{1}$ and $U_{2}$ are disjoint, then we can take $g_{1}=g_{2}=e$ in Lemma 6.1(ii). If $V:=U_{1} \cap U_{2} \neq \emptyset$, then consider the above disjoint non-empty open subsets $V_{j} \subseteq V$. By assumption, there exist $g_{1}, g_{2} \in G$ with $\alpha_{g_{j}}\left(K_{j}\right) \subseteq V_{j} \subseteq U_{j}$. Thus, the adjoint action $\sigma$ of $\alpha$ is $G$-separating.

(v) Suppose that a discrete group $G$ acts by a topologically free action $\alpha$ on a noncompact locally compact Hausdorff space $X$, and that $X$ contains more than two points. Then the crossed product $\mathrm{C}_{0}(X) \rtimes_{\sigma, \lambda} G$ is purely infinite provided that the following property holds: for any compact set $K \neq X$ and any non-empty open set $U \subseteq X$, there exists $t \in G$ such that $\alpha_{t}(K) \subseteq U$. This follows as a corollary of Theorem 1.1, also of Theorem 1.2 or of Corollary 6.2.

Proof of (v). We must verify the following properties according to each of the listed results.

(Theorem 1.1) The action $\sigma$ is exact, residually properly outer and $G$-separating.

(Theorem 1.2) The action $\sigma$ is 1-majorizing and element-wise properly outer.

(Corollary 6.2) The action $\sigma$ is exact, $G$-separating and fulfils (*): For every closed

$G$-invariant subset $Y$ of $X$ and every $g \neq e$, the set $\left\{y \in Y: \alpha_{g}(y)=y\right\}$ has empty interior.

By Remark 7.8(i), we know that the action $\sigma$ is a strong boundary (i.e., 1-majorizing) action. Hence, $A$ is $G$-simple; cf. Lemma 7.3. In particular, it follows that the action $\alpha$ on $X$ is minimal. This reduces property $(*)$ to the definition of topological freeness; cf. [1, p. 120]. The minimality of the action $\alpha$ implies that the corresponding adjoint action $\sigma: G \rightarrow \operatorname{Aut}\left(\mathrm{C}_{0}(X)\right)$ is exact, and that it becomes residually properly outer if it is element-wise properly outer. But $\sigma$ is element-wise properly outer if and only if $\alpha$ is a topological free action (see [1, p. 120] or [22, Corollary 2.22]). It remains to show that $\sigma$ is $G$-separating, but this is already contained in Remark 7.8(iv).

(vii) It is an important point that a strong boundary action is often $G$-separating and (in fact always) minimal, but the notion of a $G$-separating action is not typically related to minimality. Consequently, working with $G$-separating actions allows us to consider ideal-related classification of non-simple strongly purely infinite crossed products.

Remark 7.9. If $A$ has real rank zero, then one can restrict the conditions in Definitions 5.1, 7.1 and 7.2 to projections $p, q \in A$ in place of the elements $a, b \in A_{+}$.

Proof. Case of Definition 5.1: let $a_{1}, a_{2} \in A_{+}, c \in A, \varepsilon>0$ and define

$$
\delta:=\varepsilon /\left(1+\left\|a_{1}\right\|+\left\|a_{2}\right\|\right) .
$$

By [4], $\quad D_{j}:=\overline{a_{j} A a_{j}}$ contains an approximate unit consisting of non-zero projections. Thus, there are projections $p_{j} \in D_{j}$ such that $\left\|a_{j}-a_{j}^{1 / 2} p_{j} a_{j}^{1 / 2}\right\|<\delta$. Use [12, Proposition 2.7(i)] and the comment following [12, Proposition 2.6] to select $z_{j} \in D_{j}$ satisfying $z_{j}^{*} a_{j} z_{j}=p_{j}$. Let $c^{\prime}:=z_{1}^{*} c z_{2}$.

Suppose that there exist $e_{j} \in A, g_{j} \in G$ such that

$$
\left\|e_{j}^{*} p_{j} e_{j}-\sigma_{g_{j}}\left(p_{j}\right)\right\|<\delta \text { and }\left\|e_{1}^{*} c^{\prime} e_{2}\right\|<\delta
$$


Define $v_{j}:=\sigma_{g_{j}}\left(a_{j}^{1 / 2}\right)$ and $d_{j}:=z_{j} e_{j} \sigma_{g_{j}}\left(a_{j}^{1 / 2}\right)$. They satisfy $d_{j}^{*} a_{j} d_{j}=v_{j}^{*} e_{j}^{*} p_{j} e_{j} v_{j}$ and $v_{j}^{*} \sigma_{g_{j}}\left(p_{j}\right) v_{j}=\sigma_{g_{j}}\left(a_{j}^{1 / 2} p_{j} a_{j}^{1 / 2}\right)$. Thus, $\left\|d_{j}^{*} a_{j} d_{j}-\sigma_{g_{j}}\left(a_{j}\right)\right\|<\left(1+\left\|a_{j}\right\|\right) \delta \leq \varepsilon$. Since $d_{1}^{*} c d_{2}=v_{1}^{*} e_{1}^{*} c^{\prime} e_{2} v_{2}$, we get $\left\|d_{1}^{*} c d_{2}\right\|<\delta\left(\left\|a_{1}\right\| \cdot\left\|a_{2}\right\|\right)^{1 / 2} \leq \delta\left(\left\|a_{1}\right\|+\left\|a_{2}\right\|\right) \leq \varepsilon$.

Case of Definitions 7.1 and 7.2: let $a_{1}, a_{2} \in A_{+}$and $\varepsilon>0$, with $a_{1} \neq 0$ and $a_{2}$ not invertible in $A$ (respectively $a_{2}=1$ ). We can assume that $\varepsilon \leq 1$. Define $\delta:=\varepsilon /\left(1+\left\|a_{2}\right\|\right)$. Choose $p_{j}, z_{j} \in D_{j}:=\overline{a_{j} A a_{j}}$ as above with $\left\|a_{j}^{1 / 2} p_{j} a_{j}^{1 / 2}-a_{j}\right\|<\delta$ and $z_{j}^{*} a_{j} z_{j}=p_{j}$. Then $p_{1} \neq 0$ and $p_{2}$ is not invertible in $A$ (i.e., $p_{2} \neq 1$ ) if $a_{2}$ is not invertible, otherwise $p_{2}=1$ : if $p_{2}$ is invertible, then $1 \in a_{2} A a_{2}$, so $a_{2}$ is invertible. Conversely, if $a_{2}$ is invertible, then $\left\|p_{2}-1\right\|<\varepsilon / 2$, so $p_{2}$ is invertible.

If there are $e_{1}, \ldots, e_{n} \in A$ and $g_{1}, \ldots, g_{n} \in G$ with $\left\|p_{2}-\sum_{j} e_{j}^{*} \sigma_{g_{j}}\left(p_{1}\right) e_{j}\right\|<\delta$, then $d_{j}:=\sigma_{g_{j}}\left(z_{1}\right) e_{j} a_{2}^{1 / 2}$ satisfies $\left\|a_{2}-\sum_{j} d_{j}^{*} \sigma_{g_{j}}\left(a_{1}\right) d_{j}\right\|<\left(1+\left\|a_{2}\right\|\right) \delta \leq \varepsilon$.

Acknowledgements. Parts of this work were conducted while the second named author was at the Fields Institute from 2009 to 2012, during a stay of the authors in autumn 2011 in Copenhagen supported by Prof. M. Rørdam and during the spring of 2014 at the Fields Institute during the Thematic Program on Abstract Harmonic Analysis, Banach and Operator Algebras, 2014 supported by Prof. G. Elliott. It is a pleasure for us to forward our thanks to the Fields Institute in Toronto and the Centre for Symmetry and Deformation at the University of Copenhagen. In particular, we express our thanks for the encouragement and support from Prof. M. Rødram and Prof. G. Elliott. This research was also partially supported by the Australian Research Council.

\section{REFERENCES}

[1] R. J. Archbold and J. S. Spielberg. Topologically free actions and ideals in discrete $C^{*}$-dynamical systems. Proc. Edinb. Math. Soc. (2) 37 (1994), 119-124.

[2] G. Arzhantseva, V. Guba and M. Sapir. Metrics on diagram groups and uniform embeddings in Hilbert space. Comment. Math. Helv. 81 (2006), 911-929.

[3] E. Blanchard and E. Kirchberg. Non-simple purely infinite $C^{*}$-algebras: the Hausdorff case. J. Funct. Anal. 207 (2004), 461-513.

[4] L. G. Brown and G. K. Pedersen. $C^{*}$-algebras of real rank zero. J. Funct. Anal. 99 (1991), 131-149.

[5] J. Cuntz. K-theory for certain $C^{*}$-algebras. Ann. of Math. (2) 113 (1981), 181-197.

[6] G. A. Elliott. Some simple $C^{*}$-algebras constructed as crossed products with discrete outer automorphism groups. Publ. Res. Inst. Math. Sci. 16 (1980), 299-311.

[7] R. Exel, M. Laca and J. Quigg. Partial dynamical systems and $C^{*}$-algebras generated by partial isometries. J. Operator Theory 47 (2002), 169-186.

[8] S. Haagerup, U. Haagerup and M. Ramirez-Solano. A computational approach to the Thompson group F. Int. J. Algebra Comput. 25 (2015), 875-885.

[9] U. Haagerup and G. Picioroaga. New presentations of Thompson's groups and applications. J. Operator Theory 66 (2011), 217-232.

[10] P. Jolissaint and G. Robertson. Simple purely infinite $C^{*}$-algebras and $n$-filling actions. J. Funct. Anal. 175 (2000), 197-213.

[11] S. Kawamura and J. Tomiyama. Properties of topological dynamical systems and corresponding $C^{*}$-algebras. Tokyo J. Math. 13 (1990), 251-257.

[12] E. Kirchberg and M. Rørdam. Non-simple purely infinite $C^{*}$-algebras. Amer. J. Math. 122 (2000), 637-666.

[13] E. Kirchberg and M. Rørdam. Infinite non-simple $C^{*}$-algebras: absorbing the Cuntz algebras $\mathcal{O}_{\infty}$. Adv. Math. 167(2) (2002), 195-264. 
[14] E. Kirchberg and A. Sierakowski. Filling families and strong pure infiniteness. Preprint, 2015, arXiv:1503.08519v2.

[15] E. Kirchberg and S. Wassermann. Exact groups and continuous bundles of $C^{*}$-algebras. Math. Ann. 315 (1999), 169-203.

[16] A. Kishimoto. Outer automorphisms and reduced crossed products of simple $C^{*}$-algebras. Comm. Math. Phys. 81 (1981), 429-435.

[17] M. Laca and J. Spielberg. Purely infinite $C^{*}$-algebras from boundary actions of discrete groups. J. reine angew. Math. 480 (1996), 125-139.

[18] D. Olesen and G. K. Pedersen. Applications of the Connes spectrum to $C^{*}$-dynamical systems. III. J. Funct. Anal. 45 (1982), 357-390.

[19] G. K. Pedersen. C*-algebras and Their Automorphism Groups (London Mathematical Society Monographs, 14). Academic Press, London, 1979.

[20] J. Renault. The ideal structure of groupoid crossed product $C^{*}$-algebras, with an appendix by Georges Skandalis. J. Operator Theory 25 (1991), 3-36.

[21] M. Rørdam, F. Larsen and N. Laustsen. An Introduction to K-theory for C*-algebras (London Mathematical Society Student Texts, 49). Cambridge University Press, Cambridge, 2000.

[22] A. Sierakowski. The ideal structure of reduced crossed products. Münster J. Math. 3 (2010), 223-248. 